\title{
Development of the ReaxFF Reactive Force-Field Description of Gold Oxides
}

I. G. Shuttleworth,

School of Science and Technology,

Nottingham Trent University,

Nottingham NG11 8NS.

$\mathrm{UK}$

E-mail: ian.shuttleworth@ntu.ac.uk

\begin{abstract}
For the investigation of the interaction between oxygen and gold over length- and time-scales which reflect the operation of real catalysts the reactive force-field (ReaxFF) description of the $\mathrm{O} / \mathrm{Au}$ interaction has been developed. This development has been achieved in two stages; first, by fitting the parameters of the potential against an extensive set of both bulk and surface $\mathrm{Au}$, $\mathrm{O}_{2}$ and gold oxides derived from full quantum mechanical simulations. The resulting potential has been used in a series of molecular dynamics (MD) simulations emulating the interaction between oxygen and the $\mathrm{Au}(111)$, the missing-row $(1 \times 2)-\mathrm{mr}-\mathrm{Au}(110)$, pairing-row $(1 \times 3)$-pr$\mathrm{Au}(110)$, trenched $(1 \times 3)$-tr-Au(110) and added-row $(2 \times 1)-\operatorname{ar}-\mathrm{Au}(100)$ surfaces. These simulations have shown, in agreement with experimental studies, that oxygen diffusion from the surface to the bulk is heavily limited and generally only possible when thermal processing has started melting the Au substrate. Grand canonical Monte Carlo/molecular dynamics (GCMC/MD) simulations of Au nanoclusters have shown that the (111) and (100) facets of these particles are unreactive toward oxygen.
\end{abstract}




\section{INTRODUCTION}

Atom-scale simulations are the key to understanding how large-scale catalytic, and otherwise reactive, processes proceed. To perform an effective set of atomistic simulations a large number of initial configurations of the reactive system need to be sampled and the average reaction path can subsequently be deduced. This process, however, is computationally expensive; large configuration sets exist even for seemingly simple systems such as diatomic molecular gases reacting with well-oriented, low index crystalline surfaces. Strategies are therefore required that will reduce the computational load without reducing the configuration space. Ideally, a full set of simulations would be performed at the quantum mechanical level. Most modern Density Functional Theory (DFT) packages contain Molecular Mechanics/Molecular Dynamics (MM/MD) capabilities and can be integrated into Grand Canonical Monte Carlo (GCMC) schemes. The computational load required to do this is prohibitive even with modern highperformance computing power ${ }^{1}$. To circumvent this problem force-field techniques can be employed. In these schemes the full quantum mechanical complexity of the reacting system is replaced with an empirical or semi-empirical force field which allows large numbers of particles within and configurations of the system be simulated rapidly. This economy comes with some cost; the force field has to be 'trained' so that it describes the system in the same way as quantum mechanical simulations would. This training can be very specific to the system that is under investigation and trained force fields should only be applied to scenarios outside of their training set with careful testing. Despite these limitations it is possible, by formulating a training set which contains elements that describe in full quantum mechanical detail each configuration that can be encountered in the force-field simulation, to develop a force field which accurately and efficiently describes the system across a complete configuration set allowing reliable deductions to be drawn from the simulation set.

The reactive force-field (ReaxFF) interatomic potential ${ }^{2}$ is a method of describing the force field that acts between particles. The advantage of the ReaxFF formulation over other forcefields is that it contains bond-order/bond-length terms which describe changes in the connectivity of atoms during a reaction. This means that the energetic consequences of bonds breaking and forming are explicitly included in the force-field. The cost of including this description is an increase in the number of terms contained within the force-field. A ReaxFF field may contain a total of 20-30, or more, terms which require training. To perform this efficiently terms which describe the interactions between atoms can be grouped according to their interacting species: in the current study, therefore, terms in the training set are classified as acting between $\mathrm{Au}-\mathrm{Au}, \mathrm{Au}-\mathrm{O}$ and $\mathrm{O}-\mathrm{O}$. The $\mathrm{Au}-\mathrm{Au}$ interactions have been previously investigated $^{3}$ and the ReaxFF potential has been trained against a sequence of bulk and surface phases. However, in the current study the Au-Au interaction requires re-investigation. This is because reconstructions of the clean $\mathrm{Au}(110)$ and $\mathrm{Au}(100)$ surfaces will be used in the current study and local surface-specific changes in the Au-Au bonding will occur. In addition, the importance of relativistic terms will be evaluated. The description of the Au-O interaction terms presented in this work develops an earlier study by Joshi et al. ${ }^{4}$. Central to this development is the extension of the training set used to describe Au-O and the inclusion of higher Au-O terms in the ReaxFF field to provide a reasonable fit to the extended training set. 


\section{A. Nanoparticulate and Nanoporous Au Alloys: Reactivity and Structures}

Au is generally unreactive material ${ }^{5,6}$ in the bulk form. However, the ground-breaking studies of Haruta et al. ${ }^{7}$ revealed that gold grown in nano-particulate form undergoes significant changes in reactivity. However, nanoporous gold particles and smaller gold particle (of the order of $\mathrm{nm}$ ) have been seen to be reactive ${ }^{8,9}$ towards oxygen. Nanoporous gold particles are formed by the selective corrosion of Ag from Ag-Au alloys. Consequently they are gold-rich nanoparticles that contain pores with dimensions typically of $<100 \mathrm{~nm}$. For certain mixing ratios, e.g. $\mathrm{Ag}_{0.03} \mathrm{Au}_{0.97}{ }^{8}$, the particles are both highly selective and reactive towards oxidation. The mechanism for the reactivity is a topic of current research; contemporary studies ${ }^{10}$ have indicated that the Ag component of these alloys forms regions of locally high concentration in the presence of oxygen and the transition state during oxygen dissociation involves a significant Ag-O interaction. Similar interactions are seen during alcohol oxidation ${ }^{11,12}$.

The nanoporous particles discussed in the previous paragraph have therefore identified that the Ag component elevates the reactivity of the particle. What is not currently clear is if this elevation is due to chemical or structural effects. An enormous library of nanostructures exist $^{13,14}$ and includes Platonic solids, plates and high-index facetted structures dependent on the mechanism of surface growth, and methods of 'fingerprinting' the geometrical structures ${ }^{15}$ using the vibrational properties of the nanocluster have been developed. It is therefore challenging to broadly describe the interaction of oxygen with this class nanoparticles as the structures themselves are so diverse. Earlier studies ${ }^{16}$ have looked at the effects of reducing the Au-Au coordination number in small $(<30 \AA)$ Au particles. The small particle size and reduced metal-metal bond lengths were seen to change the hybridisation of the valence orbitals and consequently the reactivity of these particles. However the smaller size led to complications over the structure and the work discussed the presence of FCC and decahedral/icosahedral particles. The observation of range of symmetry group, 3D particles has been seen more recently for particles up to $3.5 \mathrm{~nm}^{17}$ and also planar $\mathrm{Au}_{\mathrm{n}}(\mathrm{n} \leq 11)$ flakes have been predicted using scalar-relativistic DFT calculations ${ }^{18}$. These studies have demonstrated that nanoparticulate gold structures are diverse. This complexity is enhanced by their meta-stability whereby structural transformations occur dynamically. Larger $\mathrm{Au}_{561}, \mathrm{Au}_{742}$ and $\mathrm{Au}_{923}$ clusters are 'locked'19 but others transform under electronic and temperature ${ }^{20}$ variation. These latter observations are similar to effects seen on extended crystalline surfaces where conventional surface preparation techniques under Ultra-High Vacuum (UHV) conditions include annealing to improve surface order. Though high temperature (900K) anneals are often used lower temperature anneals (350K-500K) can be effective in improving surface order. The temperature dependence is crucial as catalysts are generally operated at elevated temperatures and so the development of any theoretical description ideally should address the dependence. The studies have also highlighted the importance of the level of approximation used in describing theoretically the Au systems. The chemistry of gold, particularly in flake, cage or cluster form, requires relativistic effects to be included ${ }^{21-25}$. The reason for this is because the chemistry depends on Au s-p-d hybridisation and re-hybridisation during oxygenation. In simple terms, the large atomic number of Au makes the kinetic energy terms of the electrons within these orbitals correspondingly large and within the relativistic scheme. 


\section{B. Extended Au Surfaces: Reactivity}

Whilst a body of evidence has emerged demonstrating the effectiveness of particulate gold towards oxidation, extended single crystal Au surfaces have generally been seen to be far less reactive. Experimental attempts to overcome this limitation have included investigating the adsorption of ozone $\left(\mathrm{O}_{3}\right)$ on $\mathrm{Au}(111)^{26}$ and both high pressure $\mathrm{O}_{2}$ and $\mathrm{O}_{3}$ on $\mathrm{Au}(211)^{27}$. These procedures deposited oxygen at room temperature which could be removed by thermal processing to 515-600K though ordered overlayers were only observed for the $\mathrm{Au}(211)$ surface. In addition, other alternative oxidation routes have been demonstrated in solution: gold oxide layer formation has also been demonstrated on gold electrodes in $0.5 \mathrm{M} \mathrm{H}_{2} \mathrm{SO}_{4}{ }^{28}$ and under RF-discharge ${ }^{29}$ though these latter studies are not easily accessible to theoretical investigation. A wealth of literature exists which describes the interaction between oxygen and transition metal surfaces in general ${ }^{30}$ and generally reduces the adsorption into low energy regimes where trapping of the oxygen atom occurs before dissociation, and high energy or direct mechanisms. Mechanistically, both require electron donation from the surface to the anti-bonding orbitals of the oxygen molecule to enable dissociation. For extended gold surfaces this rate of electron transfer is low and consequently the surface resists oxidation. Though compositional and structural changes outlined above and below have been seen to effect oxidation the precise mechanism continues to remain unclear. Recent microkinetic models ${ }^{31}$ have shown that oxidation across Au nanoparticles is strongly dependent on the size of the nanoparticle and has presented some evidence that this change in reactivity is due to changes in the metallicity of the nanoparticle with size. These arguments are reflected in DFT studies of $\mathrm{Pt}_{3} \mathrm{Co}$ and $\mathrm{Pt}_{3} \mathrm{Fe}$ alloys $^{32}$ which suggested that changes in reactivity of these alloys were due to relative shifts between the d-band and the Fermi level.

The effect of roughening the $\mathrm{Au}(111)$ surface as a mechanism of enabling oxidation was investigated using $\mathrm{DFT}^{33}$ where an attractive interaction was observed to exist between oxygen atoms and vacancies on the surface. The binding energy of FCC oxygen was determined to be -2.69 to $-3.08 \mathrm{eV}$, depending on exchange-correlation functional (RPBE/PW91); additionally the values of -2.69 and $-2.78 \mathrm{eV}$ were compared when the RPBE and PBE functionals were used. The same group also investigated the effects of both oxygen dosing pressure and $\mathrm{Au}(111)$ substrate temperature ${ }^{34}$ and identified that large oxygen coverages (0.33-0.55 ML, or greater) combined with elevated surface temperatures (500-800 K, and higher) were required to form surface and selvedge located oxides. A thin selvedge-based oxide layer has been shown to be stable $^{35}$ in ab-initio studies, with oxygen migration into the bulk from the Au(111) surface, or surface oxide formation, being energetically less favoured.

The effect of steps and strain on the oxidisation of $\mathrm{Au}(111)$ surface has been investigated using $\mathrm{DFT}^{36}$. In these studies the binding energy of $\mathrm{O}_{2}$ was determined to be $-0.08,-0.15$ and -0.26 eV on $10 \%$-stretched $\mathrm{Au}(111)$, un-stretched $\mathrm{Au}(211)$ and 10\%-stretched $\mathrm{Au}(211)$, respectively. This indicated that both stepping and strain may be effective in increasing the reactivity of these surfaces towards oxidation, a conclusion which has been recently reflected in cantilever studies of (111)-textured gold surfaces in aqueous $\mathrm{H}_{2} \mathrm{SO}_{4}{ }^{37}$ which demonstrated that applying tensile strain to the surface increased the surface oxygen coverage. These conclusions were reinforced by a series of Nudged Elastic Band (NEB) simulations in the same work which showed that the activation energy of $\mathrm{O}_{2}$ dissociation was $1.37,1.12$ and $0.63 \mathrm{eV}$ on the $10 \%$ stretched $\mathrm{Au}(111)$, un-stretched $\mathrm{Au}(211)$ and 10\%-stretched $\mathrm{Au}(211)$ surfaces, respectively. 
The binding energies of atomic oxygen at selected 3-fold sites across the surfaces were determined to be -2.54 to $-3.07 \mathrm{eV}$, in agreement with studies cited earlier in this Introduction. The oxidation of $\mathrm{Au}(111)$ is consequently seen to be challenging and requires either large pressures of $\mathrm{O}_{2}$ gas or substrate temperatures, or an enabling mechanism such as surface strain or roughening, or an alternative adsorption path such as ozone adsorption.

\section{Extended Au Surfaces: Structures}

The nature of the Au-O interaction has also been investigated on $(1 \times \mathrm{r})$ reconstructions of the $\mathrm{Au}(110)$ surface $^{38}$. The work demonstrates that 'missing-row' $(1 \times 2)-\mathrm{Au}(110)$ and $(1 \times 3)-$ $\mathrm{Au}(110)$ reconstructions are stable and observed experimentally. The 'missing-row' nomenclature used in the work describes the reconstruction. However in the current work, the additional nomenclature of pairing-row (1×3)-pr-Au(110) and trench (1×3)-tr-Au(110) is used to describe the $(1 \times 3)$ reconstructions more explicitly. The work ${ }^{38}$ demonstrates that oxygen can bind stably on these reconstructions with binding energies -0.156 to $-0.349 \mathrm{eV}$. Ordered chainlike structures have been observed experimentally ${ }^{39}$ along the surface rows of Au atoms indicating that on these surface the oxygen atoms prefer to bind close to the surface rather than deeper inside the reconstructed trench or hollow. The binding energy of oxygen atoms in these structures was determined using DFT to be -3.67 to $-3.81 \mathrm{eV}^{39}$. Some discrepancies exist in the oxygen binding energies presented in work of Landmann et $\mathrm{al}^{38}$ and Hiebel et $\mathrm{al}^{39}$. However, these discrepancies may be due to the precise nature of the oxygen structure as the experimental studies have seen that oxygen chains 'straddled' the surface Au rows, rather than lying on the same side indicating a repulsive $\mathrm{O}-\mathrm{O}$ interaction.

Gold crystallises in an FCC structure. In most fundamental studies the low Miller index (111), (110) and (100) surfaces are investigated as they contain atoms of varying local coordination and are often considered as templates for more corrugated surfaces and nanoparticle facets. Gold is unusual as it exhibits reconstruction across all of these low index faces ${ }^{6,40}$. Notably it is so far the only known FCC metal that exhibits a reconstruction of its (111) surface at room temperature. Early experimental observations ${ }^{41}$ of a reconstruction of the $\mathrm{Au}(111)$ surface using Helium Atom Scattering led to 'herringbone' model of the surface $e^{42}$ and the well-known $(22 \times \sqrt{3})$ and $(23 \times \sqrt{3})$ have been investigated using DFT/PW91 and have been seen to be stable and isoenergetic, though smaller reconstructions were seen to be unstable. This is consistent with the model that the reconstruction on the $\mathrm{Au}(111)$ surface is large scale. The local reactivity ${ }^{43,44}$ of the reconstructed $\mathrm{Au}(111)$ surface shows only a small variation between surface sites with the same local symmetry (i.e. the FCC, HCP, on-top and bridge) across the reconstruction, as the registry between the surface site and the second and deeper layers vary. Consequently in the current work the only unreconstructed $\mathrm{Au}(111)$ surface will be considered as the tolerance involved in force-field approximation is anticipated to be larger than the changes in reactivity seen previously ${ }^{44}$. The validity of this approximation is reasonable in the current context where DFT simulations are used to create a training set for the ReaxFF potential, as DFT studies of $\mathrm{Au}(111)^{45}$ and relativistic DFT studies of the $\mathrm{Au}(111)$ and both $\mathrm{Pt}(111)$ and $\operatorname{Ir}(111)^{46}$ have shown that the electronic state of the reconstructed surface has only a weak dependence on the reconstruction. 
The missing row $(1 \times 2)$-mr-Au(110) and the added-row $(2 \times 1)$-ar-Au(100) surfaces have been shown to be more stable than their unreconstructed $(1 \times 1)$ counterparts ${ }^{47}$. The nature of the reconstruction has been determined experimentally ${ }^{48}$ to consist of a row of missing Au atoms along the [-1 110$]$ direction. This is accompanied by a contraction in surface layer of atoms ${ }^{49}$ and a pairing in the $2^{\text {nd }}$ and $4^{\text {th }}$ layers. Roughening has also been seen ${ }^{50}$ at $700 \mathrm{~K}$ which is suggestive that long range features of the reconstruction ${ }^{48}$ may be weak. Larger reconstructions have also been seen on the $\mathrm{Au}(110)$ surface $^{38}$. These reconstructions can shallow such as the pairing-row reconstruction where only a single row of Au atoms are removed per surface unit cell, or deeper in case of trench reconstruction where multiple rows of Au atoms are removed.

In contrast to the $\mathrm{Au}(110)$ surfaces described in the previous paragraph, the $\mathrm{Au}(100)$ surface reconstructs by increasing the surface concentration of Au atoms rather than by removing them. The added-row reconstruction results in a pseudo-hexagonal overlayer of Au atoms on an orthogonal selvedge. Recent studies ${ }^{51}$ have identified that the overlayer is composed of two rotational domains of commensurate $\mathrm{c}(28 \times 48)$ domains. The bond angle in this reconstruction is $59.75^{\circ}$ which compares with the hexagonal value of $60^{\circ}$, and the $\mathrm{Au}-\mathrm{Au}$ bond length is 0.9655 of the hexagonal layer value. The Au-O interaction is anticipated to be similar, within the tolerance of ReaxFF, to the unreconstructed $\mathrm{Au}(111)$ surface for isolated atoms consequently in the current work the training sets will be used interchangeably between the two surfaces. However, the large surface cell will be replaced by the $(5 \times 1)$ approximants which have been proven ${ }^{52}$ to capture the energetic and structural trends of the full reconstructed unit cell and have also shown to be in very close agreement with experimental models of the surface $^{47}$.

In summary, the Introduction has outlined the current interests in O/Au reactivity in terms of nanoporous and particulate Au and studies on extended Au surface. The survey has highlighted the temperature-dependent trends in the reactivity of these particles and of the extended surfaces, and has shown that relativistic effects are necessary in theoretical studies of these systems. The survey has also shown that the extended surfaces commonly investigated contain reconstructions but that in the $\mathrm{Au}(111)$ and $\mathrm{Au}(100)$ cases reasonable approximants to the reconstructions may be made. The current work will be structured in the following way: an overview of the force-field, GC-MC/MD and DFT used in the work will be given in Section II. The ReaxFF training set will be presented in Section III(A), and then the MD and GC-MC results will be presented and discussed in Sections III(B) and (C), respectively. The conclusions of the current work will then be summarised in Section IV. 


\section{THEORY AND METHODS}

\section{A. ReaxFF background}

The ReaxFF uses a description of the bond order as well as terms which describe the polarisable charge in the system to describe the reactive and the non-reactive interactions which act between systems of atoms ${ }^{2}$. The formulation in the current work was trained against a DFTgenerated set of oxide and pure $\mathrm{Au}$ and $\mathrm{O}$ configurations, the applied using a sequence of $\mathrm{MD}$ and hybrid GC-MC/MD simulations ${ }^{53}$. The Bond Order (BO) between two atoms (i,j) is written as an explicit function of the distance between pairs of atoms $\mathrm{r}_{\mathrm{ij}}$

$$
\begin{aligned}
& \mathrm{BO}_{\mathrm{ij}}=\mathrm{BO}_{\mathrm{ij}}^{\sigma}+\mathrm{BO}_{\mathrm{ij}}^{\pi}+\mathrm{BO}_{\mathrm{ij}}^{\pi \pi} \\
& =\exp \left[\mathrm{p}_{\mathrm{bo1}}\left(\frac{\mathrm{r}_{\mathrm{ij}}}{\mathrm{r}_{0}^{\sigma}}\right)^{\mathrm{p}_{\mathrm{bo} 2}}\right]+\exp \left[\mathrm{p}_{\mathrm{bo} 3}\left(\frac{\mathrm{r}_{\mathrm{ij}}}{\mathrm{r}_{0}^{\pi}}\right)^{\mathrm{p}_{\mathrm{b} 04}}\right]+\exp \left[\mathrm{p}_{\mathrm{bo5}}\left(\frac{\mathrm{r}_{\mathrm{ij}}}{\mathrm{r}_{0}^{\pi \pi}}\right)^{\mathrm{p}_{\mathrm{bo} 6}}\right]
\end{aligned}
$$

The pbo are empirical parameters and the $\mathrm{r}_{0}$ are equilibrium bond lengths. This formalism is continuous, though transitions occur between the $\sigma, \pi$ and $\pi \pi$ orbitals, and consequently generates a differentiable potential energy surface. This latter requirement is necessary for direct calculations of interatomic forces. The potential energy of the system is then written as

$\mathrm{E}_{\text {system }}=\mathrm{E}_{\text {bond }}+\mathrm{E}_{\text {angle }}+\mathrm{E}_{\text {tors }}+\mathrm{E}_{\text {over }}+\mathrm{E}_{\text {under }}+\mathrm{E}_{\text {vdWaals }}+\mathrm{E}_{\text {Coulomb }}$

Ebond is a covalent energy term which changes as covalent bonds are formed between the atoms. $E_{\text {angle }}$ and $E_{\text {tors }}$ are 3- and 4-body terms, respectively, and they describe the action of changing the angle between subtended at the centre of a group of 3 atoms or the torsional angle associated with a group of 4 atoms. $E_{\text {over }}$ and $E_{\text {under }}$ are energy penalties that reduce the over and under coordination of terms, respectively. $\mathrm{E}_{\mathrm{vdWaals}}$ and $\mathrm{E}_{\mathrm{Coulomb}}$ are the dispersive and the electrostatic interactions that act between all pairs of atoms. The empirical terms in this formulation were trained against a large set of bulk and surface configurations which were developed using DFT; the optimised force field is supplied as a supplement to this paper.

Training is performed by calculating the differences in energies between structures, e.g. between reacted and unreacted systems of extended systems and/or particles, or between strained and unstrained bulk phases ${ }^{54}$. An objective $\chi^{2}$ function can then be defined as the sum of the residuals between these energy differences and the differences between the same pairs of systems calculated using DFT. The force-field parameters were then fitted using a two-stage approach. In the first stage, a broad survey was performed of the parameter space defined by the force-field parameters to identify the positions of local minima using a Monte-Carlo-type approach $^{55}$. The local minima were the ranked and likely candidates for the global minimum 
were identified. These candidates were then fitted more accurately using linear search methods ${ }^{54}$. Only the deduced global minimum parameter is reported in the current work.

\section{B. QM background}

The training sets used in the current work were prepared using Density Functional Theory (DFT). The plane-wave DFT simulations presented in the current work were performed using the Quantum Espresso package ${ }^{56}$. For surface calculations Brillouin zone integration was performed on a $(6 \times 6 \times 1)$ grid and on either $(6 \times 6 \times 6)$ or $(10 \times 10 \times 10)$ grids for bulk studies. A first-order Methfessel-Paxton ${ }^{57}$ smearing of 0.02 Ry was used in all cases. The kinetic energy cut-offs for wave-functions and for charge density and potential were set to 50 Ry and $200 \mathrm{Ry}$, respectively. The PBE exchange-correlation functional within the Generalised Gradient Approximation (GGA) approximation was used throughout this work. The relativistic nature of the training set was defined by the choice of pseudo-potential ${ }^{58,59}$ and drawn from the Quantum Espresso pseudopotential database. Recent studies ${ }^{60}$ have shown that variations between the computational and experimental values of the lattice parameter are $<0.14 \AA$ for the transition metal pseudopotentials in this database. Using these pseudopotentials the equilibrium bulk lattice constant of FCC Au was determined to be $4.194 \AA$ which compares with the experimental value of $4.080 \AA$. The $\mathrm{O}_{2}$ molecule bond length was determined to be $1.21 \AA$ which is agreement within error with the experimental value of $1.21 \AA$.

Two DFT data sets containing structures deduced using both relativistic and non-relativistic calculations were developed in the current work. In the current work all ReaxFF's were fully trained against the non-relativistic DFT training set, and any references to the number of elements in the training set or similar refer only to the non-relativistic set. Comparisons are made between the non-relativistic and relativistic DFT sets where appropriate. Each training set then contained training elements that were either bulk $\mathrm{Au}$, bulk oxide (either $\mathrm{Au}_{2} \mathrm{O}_{3}$ or $\mathrm{Au}_{2} \mathrm{O}$ ), isolated $\mathrm{O}_{2}$, surface oxides and Nudged Elastic Band (NEB) simulations of the dissociation of molecular $\mathrm{O}_{2}$ across select low Miller index gold surfaces. The bulk phase simulations of pure $\mathrm{Au}$ and of $\mathrm{Au}_{2} \mathrm{O}_{3}$ or $\mathrm{Au}_{2} \mathrm{O}$ used single unit cells and the gas-phase $\mathrm{O}_{2}$ simulations were performed with a single $\mathrm{O}_{2}$ molecule contained in a box of dimension $10 \AA \times 10 \AA \times 10 \AA$. The oxygen binding positions on the pairing-row $(1 \times 3)$-pr-Au(110), missingrow $(1 \times 2)-m r-A u(110)$ and the $\mathrm{Au}(111)$ surfaces are shown in fig. 1. 5-7 layers/slab of $\mathrm{Au}$ atoms were used in each surface simulation depending on the degree of surface reconstruction, and subsequent slabs were separated by a distance of 10 layer spacings. The clean and oxygenated slabs were allowed to fully relax with only the central Au layer constrained.

The missing-row (1×2)-mr-Au(110) and pairing-row (1×3)-pr-Au(110) reconstructed surfaces are formed by removing rows of $\mathrm{Au}$ atoms from the unreconstructed clean $\mathrm{Au}(110)$ surface. Similarly, the $(1 \times 3)-p r-A u(110)$ surface can be converted to the trenched $(1 \times 3)$-tr-Au(110) surface by removing an additional row of surface atoms, and the resulting outermost row of second-layer Au atoms, leaving a large triangular profile when viewed along the $\left[\begin{array}{lll}0 & 0 & 1\end{array}\right]$ direction. Oxygen atoms added to the trenched $(1 \times 3)$-tr-Au(110) surface tended to migrate to the pairing-row $(1 \times 2)-\mathrm{mr}-\mathrm{Au}(110)$ positions, however, and failed to bind to positions deep within the trench. The training set elements for the trenched surfaces were consequently 
removed as their binding energies and positions were almost equivalent to those defined for the $(1 \times 2)-m r-A u(110)$ surface. Equally, the added-row $(2 \times 1)-a r-A u(100)$ training set elements were removed as the surface is hexagonal and oxygen atoms tended to bind in positions which were equivalent to those on the $\mathrm{Au}(111)$ surface.

The Nudged Elastic Band (NEB) studies were performed using 8 images per reaction pathway. To reduce the effects of lateral interactions $(4 \times 3)$ and $(4 \times 2)$ surface supercells were used respectively during every simulation involving the pairing-row reconstructed $(1 \times 3)$-pr-Au(110) and missing-row reconstructed $(1 \times 2)-m r-A u(110)$ surfaces, and a $(3 \times 3)$ surface supercell was used for simulations involving $\mathrm{Au}(111)$. The final image (adsorbed oxygen atoms) was determined by relaxing the oxygen atoms and both the surface and $2^{\text {nd }}$ layer Au atoms. The initial NEB image was at a height of 2-4 layer spacings above the outermost Au atoms. The NEB simulation was converged when the norm of the force orthogonal to the path was less than $0.05 \mathrm{ev} / \AA \AA$.

\section{RESULTS AND DISCUSSION}

\section{A. Force field development}

\section{Gold-gold metal interactions}

The $\mathrm{Au}$ atom and $\mathrm{Au}-\mathrm{Au}$ interaction parameters contained in the ReaxFF potential accompanying this paper were optimised against a training set which contained a total of 419 data points, 99 of which contained purely Au interactions. Fig. 2 shows the equations of state for bulk Au arranged in diamond, simple cubic, body-centred cubic (BCC) and face-centred cubic (FCC) structures. The reason that multiple structures are used in the current work is to increase the range of validity of the ReaxFF. This range can be further extended by applying compressive and tensile strains to the structure resulting in the curves presented in fig. 2. Each datum along these curves is an energy calculated, as indicated in the legend of the figure, either by DFT or by ReaxFF.

$E_{u c}$ is the energy of the unit cell which contains 8, 1, 2, and 4 Au atoms/unit cell in the bulk diamond, simple cubic, body-centred cubic and face-centred cubic structures, respectively. $\mathrm{V}_{\text {uc }}$ is the volume of the unit cell and increases (decreases) as tensile (compressive) strains are applied to the unit cell. When there is no applied strain then the cell is in equilibrium and the energy of the cell is Euc;eqlbrm. The strains are applied isotropically. A summary of the cohesive energies and the lattice constants for the bulk phases is shown in Table 1.

The $\mathrm{Au}$-Au interactions were also trained against the surface phases. In order to perform this training 5-7 layer Au slabs, depending on the depth of the reconstruction, were used for the unreconstructed $\mathrm{Au}(111)$ and reconstructed missing-row $(1 \times 2)-\mathrm{mr}-\mathrm{Au}(110)$, pairing-row $(1 \times 3)$-pr-Au(110), trenched $(1 \times 3)$-tr-Au(110) and added-row $(5 \times 1)$-ar-Au(100) surfaces. The ReaxFF was trained against the total DFT energy of these slabs and the resulting surface energies are shown in Table 2. The surface energy $\gamma$ is defined as 


$$
\gamma=\frac{1}{2 \mathrm{~A}}\left(\mathrm{E}_{\text {slab }}-\mathrm{nE}_{\text {atom }}\right)
$$

$E_{\text {slab }}$ is the total energy of the slab calculated using either DFT or ReaxFF, $\mathrm{n}$ is the number of $\mathrm{Au}$ atoms in the slab and $\mathrm{E}_{\text {atom }}$ is the energy per $\mathrm{Au}$ atom in the unstrained bulk FCC phase. The factor of $1 / 2$ accounts for the fact that each slab has two surfaces each of which has area A. The values in Table 2 show a close agreement between the relativistic and non-relativistic DFT estimates and this level of agreement is consistent with that seen in the equations of state for the bulk Au phases presented in fig. 2. The values of surface energy also show an off-setting of the ReaxFF values from the DFT. However, these values are reasonable: the ReaxFF values of surface energy are for the $(1 \times 3)-p r-A u(110),(1 \times 2)-m r-A u(110)$ and $(1 \times 3)$-tr-Au(110) surfaces are in close agreement with each other. This is seen amongst the DFT values as well, though the difference between the $\mathrm{Au}(110)$ surface energies and the $(5 \times 1)$-ar-Au(100) surface energy is much smaller for the DFT data sets that for the ReaxFF data set. However, the Au(111) surface energy is consistently lowest across all sequences. These results show that though there is a difference between the absolute values of the DFT and ReaxFF energies they are internally consistent. The absolute values of the surface energy of $\mathrm{Au}(111)$ is lower than the experimental value of 1.500-1.506 $\mathrm{Jm}^{-2}$ 61,and refs. therein . Improvements to DFT estimates of the surface energy can be achieved by performing Full Charge Density (FCD) calculations which has been shown to estimate the surface energy to a level of accuracy comparable to or better than that achievable with full-potential methods ${ }^{61}$ though these values still disagree by $20 \%$ with the experimental values which is a comparable error to the $30 \%$ (50\%) error seen when using full potential methods ${ }^{61}$ or in the current work, respectively. The errors in the estimates of surface energy in the DFT training sets presented in the current work are therefore expected from the level of DFT used in the current work.

\section{Gold-oxygen interactions}

The training set of the Au-O terms in the ReaxFF used both bulk and surface Au-O structures. The total Au-O and O-O components of the training set was large and contained 319 terms. The use of such an extensive training set allowed the exploration of the angular and torsional terms in the ReaxFF. Through trial and error the best weighted fit was found by focussing on the angular terms ( 3 atom centre) in the ReaxFF, and the final best fit contained 5 angular terms. The bulk training set was designed to include $\mathrm{Au}$ in low and high oxidation state; consequently the stable gold (III) oxide $\left(\mathrm{Au}_{2} \mathrm{O}_{3}\right)$ and cuprite-structured $\mathrm{Au}_{2} \mathrm{O}$ bulk oxides were considered. Fig. 3 shows the equations of state for the (a) $\mathrm{Au}_{2} \mathrm{O}_{3}$ and (b) $\mathrm{Au}_{2} \mathrm{O}$ bulk oxides derived from relativistic and non-relativistic DFT, and using the fully trained ReaxFF potential. In both cases the bulk unit cells were orthogonal and contained a total of either $40\left(\mathrm{Au}_{2} \mathrm{O}_{3}\right)^{62}$ or $6\left(\mathrm{Au}_{2} \mathrm{O}\right)^{63}$ atoms. The fractional coordinates of the $\mathrm{Au}$ and $\mathrm{O}$ atoms within the $\mathrm{Au}_{2} \mathrm{O}_{3}$ unit cell were fixed at their experimentally determined values ${ }^{62}$ with no further relaxation attempted.

The equilibrium lattice parameters of the $\mathrm{Au}_{2} \mathrm{O}_{3}$ system were determined to be 13.08 (13.04), 10.78 (10.72) and 4.02 (3.99) $\AA$ using non-relativistic (relativistic) DFT, and were comparable to the experimental values of $12.827,10.520$ and $3.838 \AA$, respectively ${ }^{62}$. These parameters were obtained using the procedure outlined $\mathrm{in}^{63}$. The heats of formation of the equilibrium $\mathrm{Au}_{2} \mathrm{O}_{3}$ and $\mathrm{Au}_{2} \mathrm{O}$ bulk phases were calculated to be -0.478 and $0.123 \mathrm{eV}$ respectively, using 
the optimised ReaxFF presented in the current work, which compares with values of -0.52 and $0.228 \mathrm{eV}$, respectively, which were calculated using non-relativistic PBE-GGA DFT. The variations in the volume of the unit cell required to obtain the equation of state for the $\mathrm{Au}_{2} \mathrm{O}_{3}$ system presented in fig. 3 (a) were achieved by straining the unit cell along the $b$ axis. The volume of the unit cell is $\mathrm{V}_{\mathrm{uc}}=\mathrm{abc}$ and the energy per unit cell is evaluated across all 40 atoms (24 $\mathrm{O}$ and $16 \mathrm{Au}$ ) within the unit cell. For the $\mathrm{Au}_{2} \mathrm{O}$ system the atoms were held in the cuprite positions $^{63}$ and the equilibrium lattice constant was determined to be 4.84 (4.79) $\AA$ using nonrelativistic (relativistic) DFT which are comparable to previously determined values of

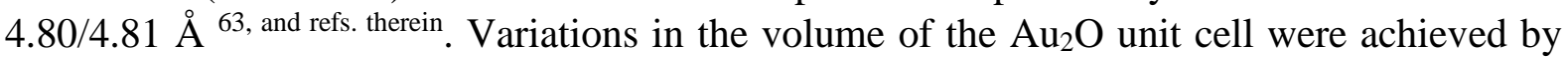
straining all 3 of the orthogonal crystal axes equally and the volume of the unit cell is $V_{u c}=a^{3}$.

The differences in the absolute values of the energies per unit cell $\mathrm{E}_{\mathrm{uc}}$ between the relativistic and non-relativistic training sets arose because differences in the Kohn Sham energies of the single $\mathrm{Au}$ and $\mathrm{O}$ atoms, which were magnified particularly in the case of $\mathrm{Au}_{2} \mathrm{O}_{3}$ because of the large number of atoms present. Fig. 4 shows the binding energy $\mathrm{E}_{\mathrm{O}_{2}}$ of the isolated oxygen molecules

$\mathrm{E}_{\mathrm{O}_{2}}=\frac{1}{2} \mathrm{E}_{\mathrm{M} ; \mathrm{O}_{2}}-\mathrm{E}_{\mathrm{O}}$

$\mathrm{E}_{{\mathrm{M} ; \mathrm{O}_{2}}_{2}}$ is the total energy of the isolated $\mathrm{O}_{2}$ molecule and $\mathrm{E}_{\mathrm{O}}$ is the energy of an isolated oxygen atom. The absolute value of the $\mathrm{E}_{\mathrm{O}_{2}}$ was $-4.27(-4.24) \mathrm{eV}$ for the non-relativistic and relativistic DFT training sets, respectively, which compares to the experimental value of $5.15 \mathrm{eV}$ and with the ReaxFF value of $-4.35 \mathrm{eV}$. This demonstrates that the importance of relativistic corrections to the isolated $\mathrm{O}_{2}$ molecule was minor, and as the $\mathrm{O}_{2}$ equation of state was only required in non-relativistic form for the training set, the relativistic equation of state is not included in the current work.

The final evaluation of the relativistic correction in the current work is presented in fig. 5 which shows the isolated oxygen atom binding energies for the $\mathrm{O} /(1 \times 3)-\mathrm{pr}-\mathrm{Au}(110), \mathrm{O} /(1 \times 2)-\mathrm{mr}-$ $\mathrm{Au}(110), \mathrm{O} /(3 \times 3)-\mathrm{Au}(111)$. In this work, $1 \mathrm{ML}$ is defined as a surface coverage equal to the number of $\mathrm{Au}$ atoms in an unreconstructed, ideally bulk-terminated $\mathrm{Au}(111)$ and $\mathrm{Au}(110)$ surface. The binding energies in figs. 5 and 6 are defined by

$\mathrm{E}=\frac{1}{\mathrm{n}}\left(\mathrm{E}_{\mathrm{O}_{\mathrm{n}} / \mathrm{Au}}-\mathrm{E}_{\mathrm{Au}}-\frac{\mathrm{n}}{2} \mathrm{E}_{\mathrm{Eqm}_{\mathrm{O}_{2}}}\right)$

$\mathrm{E}_{\mathrm{O}_{\mathrm{n}} / \mathrm{Au}}, \mathrm{E}_{\mathrm{Au}}$ and $\mathrm{E}_{\mathrm{Eqm} ; \mathrm{O}_{2}}$ are the total energies of the oxygenated Au slab, the clean Au slab and the isolated, unstrained $\mathrm{O}_{2}$ molecule, respectively, and $\mathrm{n}$ is the total number of oxygen atoms 
populating the slab. In fig. 5 (a) the binding energies across the $\mathrm{O} /(1 \times 3)-p r-\mathrm{Au}(110)$ are presented. The abscissa labels denote the binding position defined in fig. 1 (a). In this system positions $d$ and c ( $f$ and $g$ ) were metastable and consequently only one of the sets of binding energies are presented. The $d$ and e/c ( $f$ and g) sites were similarly metastable on the $O /(1 \times 2)$ $\mathrm{mr}-\mathrm{Au}(110)$ surface and fig. 5 (b) is similarly truncated. No equivalent effect was noted for the $\mathrm{Au}(111)$ surface in fig. 5 (c) though the FCC and HCP were noted to be energetically very similar. Across all three surfaces the on-top position was noted to be energetically unfavourable. The magnitudes of the binding energy for the lay broadly in the region -2.2 to $-3 \mathrm{eV}$ which is consistent with previous reports on this surface ${ }^{33}$ and were discussed in the Introduction to this work. A similar order agreement was noted for the oxygen atoms bound to the $\mathrm{Au}(110)$ surfaces though the previous reports ${ }^{38,39}$ for the missing row surfaces carry a nominal error bar of $\pm 1 \mathrm{eV}$ as outlined in the Introduction.

The results in fig. 5 for isolated oxygen adsorption on reconstructed surfaces and the in figs. 2 and 3 both the pure metal and bulk oxide studies, and in Table 2 for the surface energy studies, have shown that the ReaxFF potential can be fitted to the non-relativistic DFT set. The results in figs. 2, 3 and 5 and Table 2 have also shown that trends seen in the non-relativistic DFT such as the positions of the minima of the equations of state in figs. 2 and 3 or the variation between stable $(\mathrm{E}<0)$ and unstable $(\mathrm{E}>0)$ binding positions presented in fig. 5 - are reflected in the relativistic DFT results. Only the ReaxFF fit to the non-relativistic DFT set and only nonrelativistic DFT results will be considered in the remainder of this work.

In order to include pairwise oxygen-oxygen interactions and to add a set of elements that realistically describe these interactions during dissociation a series of Nudged Elastic Band (NEB) simulations were performed across the reconstructed Au(110) surface. NEB studies have been performed previously to describe $\mathrm{O}_{2}$ dissociation over the unstrained and strained $\mathrm{Au}(111)$ and $\mathrm{Au}(211)$ surfaces ${ }^{36}$. Binding energies of $\mathrm{O}_{2}$ of $-0.08,-0.15$ and $-0.26 \mathrm{eV}$ were determined on the 10\%-stretched $\mathrm{Au}(111)$, unstretched $\mathrm{Au}(211)$, and 10\%-stretched $\mathrm{Au}(211)$, respectively. The study ${ }^{36}$ also investigated the binding energies of oxygen atoms on these surfaces and showed that these energies were $2-3 e V$ greater than the $\mathrm{O}_{2}$ binding energies.

To initiate the investigation a sequence of structures containing 2 oxygen atoms per unit cell were simulated using DFT. These structures are shown in fig. 6 for the $(1 \times 3)$-pr-Au(110) and $(1 \times 2)-m r-A u(110)$. Both surfaces showed that the strongest O-Au bond occurs when the oxygen atoms 'straddle' the reconstructed rows of Au atoms. This is in perfect agreement with the experimental studies of these systems $s^{38,39}$ and this trend is also reflected in the ReaxFF description of the system shown in fig. 6 . These observations highlight the central role played by the step and discontinuities during these dissociation processes and are highly suggestive that very similar phenomena are likely to be seen investigating the dissociation processes on either of the reconstructed $\mathrm{Au}(110)$ surface. The training set consequently contained NEB paths whose final binding positions were set at several orientations across the step of the $(1 \times 3)$-pr$\mathrm{Au}(110)$ surface and straddling the reconstructed rows on the $(1 \times 2)-\mathrm{mr}-\mathrm{Au}(110)$ surface. These NEB pathways are presented in figs. 7 and 8, respectively. In these figures the ReaxFF energies were obtained by applying the trained ReaxFF to the DFT-NEB structures. By comparing the binding energies in figs. 6 and the dissociation energies in figs. 7 and 8 the dissociation energies are seen to be $2-2.5 \mathrm{eV}$ less than the binding energies which is in strong agreement with the trend seen in previous work ${ }^{36}$ and discussed earlier. This trend is also seen internally within both the ReaxFF and DFT training sets, showing that though the absolute values of the binding 
and dissociation energies are different the qualitative trends predicted by both ReaxFF and DFT are very similar. Along the reaction pathways shown in Figs. 7 (a-d) activation energies for oxygen association of between 0.687 and $0.818,1.174$ and 2.526, 1.272 and 2.310 and 0.920 and $1.851 \mathrm{eV}$ were calculated, respectively, using the optimised ReaxFF presented in the current work, and 0.687 and $0.818,1.423$ and 2.879, 1.272 and 2.310, and 0.920 and $1.698 \mathrm{eV}$ were calculated, respectively, using non-relativistic DFT. Along the reaction pathways shown in Figs. 8 (a-b) activation energies for oxygen association of 1.082 and $1.494 \mathrm{eV}$ were calculated, respectively, using the both optimised ReaxFF presented in the current work and non-relativistic DFT.

\section{B. MD simulations of the surface reactivity of gold}

Fig. 9 shows a summary of a series of temperature ramped NVT simulations of $\mathrm{O}_{2}$ cracking on the (1×3)-pr-Au(110), (1×2)-mr-Au(110), Au(111), (1×3)-tr-Au(110), and (5×1)-ar-Au(100) surfaces. The simulations were performed by arranging oxygen molecules at distances of 1-2 layer spacings above the outermost Au layers for the $\mathrm{Au}(111)$ and $\mathrm{Au}(110)$ surfaces. The molecules were arranged in either in a planar arrangement for simulations over the Au(111) surfaces or along a sawtooth profile for simulations over the Au(110) surfaces and then allowing the system to fully relax before starting the simulation. Because of the strong similarities between the structures of the hexagonal $\mathrm{Au}(111)$ and $(5 \times 1)$-ar-Au(100) layers, the oxygen molecules were placed within the surface layer of the $\mathrm{Au}(100)$ surface to allow a comparative study.

The analysis in fig. 9 indicates the number of oxygen molecules that above - $\mathrm{O}_{2}$ (surf) - or below - $\mathrm{O}_{2}$ (selv) - the surface plane, where 'above' and 'below' denote directions away from and towards the bulk, respectively, as well as the number of oxygen atoms - $\mathrm{O}$ atom - that are not part of an $\mathrm{O}_{2}$ molecule. This condition was defined as when the O-O bond length is $>1.5$ A. Fig. 9 shows that oxygen tend to remain in molecular form and above the surface, and require significant thermal activation to either crack or become absorbed into the surface. This is in good agreement with the experimental evidence for these systems outlined in the Introduction. The absorbed oxygen component of the $(5 \times 1)$-ar- $\mathrm{Au}(100)$ is also seen to migrate above the surface without cracking at temperatures below $1000 \mathrm{~K}$. This shows that significant thermal activation is required before absorption can stably exist even for the case of implanted oxygen molecules.

Fig. 10 shows the Au component of the temperature ramped simulations and shows that significant thermal disruption of the Au layers occurs at temperatures of approximately $2000 \mathrm{~K}$ for the (1×3)-pr-Au(110), (1×2)-mr-Au(110), $\mathrm{Au}(111)$ and $(1 \times 3)$-tr-Au(110) surfaces, and at approximately $1800 \mathrm{~K}$ for the $(5 \times 1)$-ar-Au(100) surface. For comparison, the current simulations were repeated using a previously proposed O-Au ReaxFF potential ${ }^{4}$ which predicted surface melting temperatures of approximately $2000 \mathrm{~K}$ for all five surfaces.

The onset of this melting can be correlated with the appearance of a population of selvedge and bulk oxygen evidenced by the $\mathrm{O}_{2}$ (selv) parameter presented in fig. 9. The onset of permanent increases to $\mathrm{O}_{2}$ (selv) began at 1900, 1800, 2200 and $1600 \mathrm{~K}$ for the $(1 \times 3)$-pr-Au(110), $(1 \times 2)$ mr-Au(110), $\mathrm{Au}(111)$ and (1×3)-tr-Au(110) surfaces, respectively. These observations show 
that the Au surfaces are unreactive at low temperatures towards molecular oxygen. The surface can be activated thermally towards oxygen but the temperature at which this activation occurs depends on the surface structure, with the highly corrugated (1×3)-tr-Au(110) surface showing the lowest temperature activation and the smooth $\mathrm{Au}(111)$ surface showing the highest.

These observations suggest that the a transition will occur at surface temperatures $1500-2000 \mathrm{~K}$ depending on the structure of the surface. To investigate the behaviour of the O-Au and AuAu components across this transition a sequence of isothermal simulations were performed at $1500 \mathrm{~K}$ and $2000 \mathrm{~K}$; these temperatures were selected as the lower (higher) is sufficient to study changes in the $\mathrm{O}-\mathrm{Au}(\mathrm{Au}-\mathrm{Au})$ components of the system. For these simulations oxygen atoms were arranged in a similar initial structure to those used for the temperature ramped studies presented in figs. 9 and 10 for the (1×3)-pr-Au(110), (1×2)-mr-Au(110), Au(111) and (1×3)tr-Au(110) surface. However, the oxygen molecules were arranged at height of 1 layer spacing above the (5×1)-ar- $\mathrm{Au}(100)$ surface for these isothermal studies.

The results of these isothermal simulations are shown in fig. 11. For the higher temperature a bi-modal behaviour is seen whereby the oxygen atoms either desorb from the surface or become incorporated in the surface Au layer. This type of behaviour has been seen in other investigations of oxide formation on $\mathrm{Au}^{35}$ where the process of oxidation was shown to be kinetically limited. This bimodal behaviour is accompanied by thermal disruption of the $\mathrm{Au}$ layers and is consistent with the model developed in the temperature ramped component of this section which identified thermal disruption of the Au atoms as necessary for incorporation of oxygen into the selvedge and bulk regions of the crystal. This model is also consistent with the low temperature $(1500 \mathrm{~K})$ simulations presented in fig. 11 . In these simulations the surface oxygen is seen to either desorb or to remain on the surface, notably on $(5 \times 1)$-ar-Au(100). In these low temperature simulations little disruption of the Au atoms can be seen.

\section{Grand canonical Monte Carlo simulations of gold oxide formation}

The structure of small gold nanoparticles is a diverse field and was outlined in the Introduction to this work. Consequently the current study will investigate the behaviour of small, ideally bulk-terminated FCC nanoparticles using GC-MC/MD. The nanoparticles contained either 43, 135 or $249 \mathrm{Au}$ atoms and the surfaces contained (111) and (100) facets. Using this approach the importance of ensemble effects and the relative importance of thermal activation can be investigated. Following the extended surface investigations presented in the previous section and the previously identified meta-stability of gold clusters ${ }^{19,20}$ the nanoparticle investigations in this section were performed at 500, 1500, 2000 and $2200 \mathrm{~K}$ and using oxygen chemical potentials of $-109.23,-138.17,-153.92$ and $-157.11 \mathrm{kcal} / \mathrm{mol}$, respectively ${ }^{53}$.

The studies showed that even after extensive (>250000) GC-MC/MD iterations the nanoparticles remained unreacted with oxygen accounting for less than $1 \%$ total number of atoms in each simulation. For the $500 \mathrm{~K}$ and the $1500 \mathrm{~K}$ simulations these observations indicate that reducing the size of the (111) and (100) facets does not increase their reactivity towards oxygen. For the higher temperature $2000 \mathrm{~K}$ and $2200 \mathrm{~K}$ simulations the observations indicate that thermal activation alone is insufficient to enable bulk oxide formation or cracking of the oxygen molecule. The final reacted structures for the $2000 \mathrm{~K}$ simulations are shown in Fig. 12 
and demonstrate the very low reactivity of the nanoparticles towards oxygen. The experimental observations of the reactivity of these nanoparticles towards oxygen outlined in the Introduction are therefore not purely explained by the Au-O interaction within ReaxFF and are consequently predicted to be due to other interactions such as support effects.

\section{CONCLUSIONS}

The current work has developed the reactive force-field (ReaxFF) description of the O-Au interaction. This development has been achieved through the development of a large DFT structure set composed of both bulk and surface Au and O/Au phases. Structural and energetic trends are mirrored between relativistic and non-relativistic components of the DFT data set. Application of the trained ReaxFF to oxidation process on both extended (1×3)-pr-Au(110), $(1 \times 2)-m r-A u(110), A u(111),(1 \times 3)-t r-A u(110)$, and $(5 \times 1)-a r-A u(100)$ surfaces has shown that bulk oxide formation is kinetically limited and requires large thermal activation close to the melting point of the surface to proceed. Small Au nanoparticles composed of (111) and (100) facets are predicted to be unreactive towards oxygen.

\section{ACKNOWLEDGMENTS}

I would like to thank Prof. Adri Van Duin and Dr. Thomas Senftle (Pennsylvania State University) for their provision of the ReaxFF and GC/MC-MD codes, and their advice over its implementation. This research used the computational resources of the Supercomputing Laboratory at the King Abdullah University of Science \& Technology (KAUST) in Thuwal, Saudi Arabia. 


\section{REFERENCES}

(1) Woodley, S. M.; Catlow, C. R. A. High performance computing in the chemistry of materials. Phys. Chem. Chem. Phys. 2014, 16(39), 21001-21001.

(2) Senftle, T. P.; Hong, S.; Islam, Md. M.; Kylasa, S. B.; Zheng, Y.; Shin, Y. K.; Junkermeier, C.; Engel-Herbert, R.; Janik, M. J.; Aktulga, H. M., et al. The ReaxFF reactive force-field: development, applications and future directions. npj Comptational Materials 2016, 2, 15011.

(3) Keith, J. A.; Fantauzzi, D.; Jacob, T. Reactive forcefield for simulating gold surfaces and nanoparticles. Phys. Rev. B. 2010, 81, 235404.

(4) Joshi, K.; van Duin, A. C. T.; Jacob, T. Development of a ReaxFF description of gold oxides and initial application to cold welding of partially oxidized gold surfaces. $J$. Mater. Chem. 2010, 20, 10431-10437.

(5) Mavrikakis, M.; Stoltze, P.; Nørskov, J. K. Making gold less noble. Cat. Letts. 2000, 64, 101-106.

(6) Meyer, R.; Lemire, C.; Shaikhutdinov, Sh. K.; Freund, H.-J. Surface chemistry of catalysis by gold. Gold Bull. 2004, 37/1-2, 72-124.

(7) Haruta, M.; Kobayashi, T.; Yamada, H. S. N. Novel gold catalysts for the oxidation of carbon monoxide at a temperature far below $0^{\circ} \mathrm{C}$. Chem. Lett. 1987, 2, 405-408.

(8) Biener, J.; Biener, M. M.; Madix, R. J.; Friend, C. M. Nanoporous gold: understanding the origin of the reactivity of a 21st century catalyst made by preColumbian technology. ACS Catal. 2015, 5(11), 6263-6270.

(9) Personick, M. L.; Montemore, M. M.; Kaxiras, E.; Madix, R. J.; Biener, M. M.; Friend C. M. Catalyst design for enhanced sustainability through fundamental surface chemistry. Philos. Trans. R. Soc., Ser. A 2016, 374, 20150077.

(10) Montemore, M. M.; Madix, R. J.; Kaxiras, E. How does nanoporous gold dissociate molecular oxygen. J. Phys. Chem. C. 2016, 120, 16636-16640.

(11) Wang, L.-C.; Personick, M. L.; Karakalos, S.; Fushimi, R.; Friend, C. M.; Madix, R. J. Active sites for methanol partial oxidation on nanoporous gold catalysts. J. Catal. 2016, 344, 778-783.

(12) Wang, L.-C.; Friend, C. M.; Fushimi, R.; Madix R. J. Active site densities , oxygen activation and adsorbed reactive oxygen in alcohol activation on npAu catalysts. Faraday Discuss. 2016, 188, 57-67.

(13) Zhang, J.; Langille, M. R.; Personick, M. L.; Zhang, K.; Li, S.; Mirkin, C. A. Concave cubic gold nanocrystals with high index facets. J. Am. Chem. Soc. 2010, 132, 1401214014.

(14) Langille, M. R.; Personick, M. L.; Zhang, J.; Mirkin, C. A. Defining rules for the shape evolution of gold nanoparticles. J. Am. Chem. Soc. 2012, 134, 14542-14554. 
(15) Sauceda, H. E.; Garzón, I. L. Vibrational properties and specific heat of core-shell Au-Au icosahedral nanoparticles. Phys. Chem. Chem. Phys. 2015, 17, 28054-28059.

(16) Miller, J. T.; Kropf, A. J.; Zha, Y.; Regalbuto, J. R.; Delannoy, L.; Louis, C.; Bus, E.; van Bokhoven, J. A. The effect of gold particle size on Au-Au bond length and reactivity toward oxygen in supported catalysts. J. Catal. 2006, 240, 222-234.

(17) Li, H.; Li, L.; Pedersen, A.; Gao, Y.; Khetrapal, N.; Jónsson, H.; Zeng, X. C. Magicnumber gold nanoclusters with diameters from 1 to $3.5 \mathrm{~nm}$ : relative stability and catalytic activity for CO oxidation. Nano Lett. 2015, 15, 682-688.

(18) Fernández, E. M.; Soler, J. M.; Balbás, L. C. Planar and cagelike structures of gold clusters: Density-functional pseudopotential calculations. Phys. Rev. B 2006, 73, 235433.

(19) Wells, D. W.; Rossi, G.; Ferrando, R.; Palmer, R. E. Metastability of the atomic structures of size-selected gold nanoparticles. Nanoscale 2015, 7, 6498-6503.

(20) Ali, S.; Myasnichenko, V. S.; Neyts, E. C. Size-dependent strain and surface energies of gold nanoclusters. Phys. Chem. Chem. Phys. 2016, 18, 792-800.

(21) Häkkinen, H. Atomic and electronic structure of gold clusters: understanding flakes, cages and superatoms from simple concepts. Chem. Soc. Rev. 2008, 37, 1847-1859.

(22) Pyykkö, P. Theoretical chemistry of gold. Angew. Chem. Int. Ed. 2004, 43, 44124456.

(23) Pyykkö, P. Theoretical chemistry of gold. II. Inorg. Chim. Acta 2005, 358, 41134130.

(24) Jones, T. E.; Piccinin, S.; Stampfl, C. Relativity and the nobility of gold. Mater. Chem. Phys. 2013, 141, 14-17.

(25) Huang, W.; Ji, M.; Dong, C.-D.; Gu, X.; Wang, L.-M.; Gong, X. G.; Wang, L.-S. Relativistic effects and the unique low-symmetry structures of gold nanoclusters. ACS Nano. 2008, 2(5), 897-904.

(26) Saliba, N.; Parker, D. H.; Koel, B. E. Adsorption of oxygen in Au(111) by exposure to ozone. Surf. Sci. 1998, 410, 270-282.

(27) Kim, J.; Samano, E.; Koel, B. E. Oxygen adsorption and oxidation reactions on $\mathrm{Au}(211)$ surfaces: Exposures using $\mathrm{O}_{2}$ at high pressures and ozone $\left(\mathrm{O}_{3}\right)$ in UHV. Surf. Sci. 2006, 600, 4622-4632.

(28) Juodkazis, K.; Juodkazytė, J.; Jasulitienè, V.; Lukinskas, A.; Šebeka, B. XPS studies on the gold oxide surface layer formation. Electrochem. Commun. 2000, 2, 503-507. 
(29) Kibis, L. S.; Stadnichenko, A. I.; Koscheev, S. V.; Zaikovskii, V. I.; Boronin, A. I. Highly oxidised gold nanoparticles: in situ synthesis, electronic properties, and reaction probability toward CO oxidation. J. Phys. Chem. C. 2014, 119, 2523-2529.

(30) Nolan, P. D.; Clay Wheeler, M.; Davis, J. E.; Buddie Mullins, C. Mechanisms of initial dissociative chemisorption of oxygen on transition-metal surfaces. Acc. Chem. Res. 1998, 31, 798-804.

(31) Roldán, A.; Novell, G.; Ricart, J. M.; Illas, F. Theoretical simulation of temperature programmed desorption of molecular oxygen in isolated Au nanoparticles from density functional calculations and microkinetics models. J. Phys. Chem. C. 2010, 114, 5101-5106.

(32) Xu, Y.; Ruban, A. V.; Mavrikakis, M. Adsorption and dissociation of $\mathrm{O}_{2}$ on Pt-Co and Pt-Fe alloys. J. Am. Chem. Soc. 2004, 126, 4717-4725.

(33) Baker, T. A.; Friend, C. M.; Kaxiras, E. Atomic oxygen adsorption on Au(111) surfaces with defects. J. Phys. Chem. C. 2009, 113, 3232-3238.

(34) Baker, T. A.; Xu, B.; Liu, X.; Kaxiras, E.; Friend, C. M. Nature of oxidation of the Au(111) surface: experimental and theoretical tnvestigation. J. Phys. Chem. C. 2009, 113, 16561-16564.

(35) Shi, H.; Stampfl, C. First-principles investigations of the structure and stability of oxygen adsorption and surface oxide formation at Au(111). Phys. Rev. B 2007, 76, 075327.

(36) Xu Y.; Mavrikakis, M. Adsorption and dissociation of $\mathrm{O}_{2}$ on gold surfaces: effect of steps and strain. J. Phys. Chem. B. 2003, 107, 9298-9307.

(37) Deng, Q.; Gopal, V.; Weissmüller, J. Less noble or more noble: how strain affects the binding of oxygen on gold. Angew. Chem. Int. Ed. 2015, 54, 12981-12985.

(38) Landmann, M.; Rauls, E.; Schmidt, W. G. Chainlike Au-O structures on Au(110)$(1 \times r)$ surfaces calculated from first principles. J. Phys. Chem. C. 2009, 113, 56905699.

(39) Hiebel, F.; Montemore, M. M.; Kaxiras, E.; Friend, C. M. Direct visualisation of quasi-ordered oxygen chain structures on Au(110)-(1×2). Surf. Sci. 2016, 650, 5-10.

(40) J. Gong. Structure and surface chemistry of gold-based model catalysts. Chem. Rev. 2011, 112, 2987-3054.

(41) Harten, U.; Lahee, A. M.; Toennies, J. P.; Wöll, Ch. Observation of a soliton reconstruction of $\mathrm{Au}(111)$ by high-resolution helium-atom diffraction. Phys. Rev. Lett. 1985, 54(24), 2619-2622.

(42) Narasimhan, S.; Vanderbilt, D. Elastic stress domains and the herringbone reconstruction on Au(111). Phys. Rev. Lett. 1992, 69(10), 1564-1567. 
(43) Torres, E.; DiLabio, G. A. A density functional theory study of the reconstruction of gold (111) surfaces. J. Phys. Chem. C. 2014, 118, 15624-15629.

(44) Hanke, F.; Björk, J. Structure and local reactivity of the Au(111) surface reconstruction. Phys. Rev. B 2013, 87, 235422.

(45) Seitsonen, A. P. Electronic structure of reconstructed Au(111) studied with density functional theory. Surf. Sci. 2016, 643, 150-155.

(46) Dal Corso, A. Clean $\operatorname{Ir}(111)$ and Pt(111) electronic surface states: A first-principle fully relativistic investigation. Surf. Sci. 2015, 637-638, 106-115.

(47) Shi, H.; Stampfl, C. Shape and surface structure of gold nanoparticles under oxidizing conditions. Phys. Rev. B 2008, 77, 094127.

(48) Robinson, I. K. Direct determination of the $\mathrm{Au}(110)$ reconstructed surface by x-ray diffraction. Phys. Rev. Lett. 1983, 50(15), 1145-1148.

(49) dos Reis, D. D.; Negreiros, F. R.; de Carvalho, V. E.; Soares, E. A. Geometry of the Au(110)-(1×2) missing-row clean surface: A new LEED and DFT study. Surf. Sci. 2010, 604, 568-573.

(50) Cvetko, D.; Lausi, A.; Morgante, A.; Tommasini, F.; Prince, K. C. He beam study of deconstruction and roughening of $\mathrm{Au}(110)(1 \times 2)$. Surf. Sci. 1992, 269-270, 68-73.

(51) Hammer, R.; Sander, A.; Förster, S.; Kiel, M.; Meinel, K.; Widdra, W. Surface reconstruction of $\mathrm{Au}(001)$ : High-resolution real-space and reciprocal-space inspection. Phys. Rev. B 2014, 90, 035446.

(52) Havu, P.; Blum, V.; Havu, V.; Rinke, P.; Scheffler, M. Large-scale surface reconstruction energetics of $\mathrm{Pt}(100)$ and $\mathrm{Au}(100)$ by all-electron density functional theory. Phys. Rev. B 2010, 82, 161418(R).

(53) Senftle, T. P.; Meyer, R. J.; Janik, M. J.; van Duin, A. C. T. Development of a ReaxFF potential for Pd/O and application to palladium oxide formation. J. Chem. Phys. 2013, 139(4), 044109-1/14.

(54) van Duin, A. C. T.; Baas, J. M. A.; van de Graaf, B. Delft molecular mechanics: a new approach to hydrocarbon force fields. Inclusion of a geometry-dependent charge calculation. J. Chem. Soc., Faraday Trans. 1994, 90, 2881-2895.

(55) Press, W. H.; Flannery, B. P.; Teukolsky, S. A.; Vetterling, W. T. Numerical Recipes in C; Cambridge University Press: Cambridge, U.K., 2002. 
(56) Giannozzi, P.; Baroni, S.; Bonini, N.; Calandra, M.; Car, R.; Cavazzoni, C.; Ceresoli, D.; Chiarotti, G. L.; Cococcioni, M.; Dabo, I., et al. QUANTUM ESPRESSO: a modular and open-source software project for quantum simulations of materials. $J$. Phys. Condens. Matter 2009, 21, 395502-1/19.

(57) Methfessel, M.; Paxton, A. T. High-precision sampling for Brillouin-zone integration in metals. Phys. Rev. B 1989, 40, 3616-3621.

(58) The Au.pbe-mt fhi.UPF and O.pbe-mt fhi.UPF pseudopotentials were used for the non-relativistic simulations and the Au.rel-pbe-n-kjpaw_psl.1.0.0.UPF and O.rel-pben-kjpaw_psl.1.0.0.UPF pseudopotentials were used for the relativistic simulations. All pseudopotentials were taken from the Quantum ESPRESSO pseudopotential data base: http://www.quantum-espresso.org/pseudopotentials.

(59) Dal Corso, A. Pseudopotentials periodic table: From H to Pu. Comput. Mater. Sci. 2014, 95, 337-350.

(60) Shuttleworth, I. G.. Non-linear modelling of the effects of strain on transition metal surfaces. Chem. Phys. Lett. 2016, 666, 51-57.

(61) Vitos, L.; Ruban, A. V.; Skriver, H. L.; Kollár, J. The surface energy of metals. Surf. Sci. 1998, 411, 186-202.

(62) Jones, P. G.; Rumpel, H.; Schwarzmann E.; Sheldrick, G. M. Gold(III) oxide. Acta. Cryst. B 1979, 35, 1435-1437.

(63) Shi, H.; Asahi, R.; Stampfl, C. Properties of the gold oxides $\mathrm{Au}_{2} \mathrm{O}_{3}$ and $\mathrm{Au}_{2} \mathrm{O}$ : First principles investigation. Phys. Rev. B 2007, 75, 205125-1/8. 
Table 1. Cohesive energies $\left(E_{c}\right)$ and equilibrium lattice parameters $\left(a_{0}\right)$ for the diamond, simple cubic, body-centred cubic (BCC) and face-centred cubic (FCC) structures of Au. The 'ReaxFF' and 'DFT' columns were calculated using the optimised ReaxFF force field presented in the current work and non-relativistic PBE-GGA DFT simulations, respectively.

\begin{tabular}{|l|l|l|l|l|}
\hline \multirow{2}{*}{ Structure } & \multicolumn{2}{|c|}{$\mathrm{E}_{\mathrm{c}}(\mathrm{eV})$} & \multicolumn{2}{c|}{$\mathrm{a}_{0}(\AA)$} \\
\cline { 2 - 5 } & ReaxFF & DFT & ReaxFF & DFT \\
\hline Diamond & 2.77 & 2.91 & 6.72 & 6.25 \\
\hline Simple cubic & 3.08 & 3.53 & 2.95 & 2.80 \\
\hline BCC & 3.63 & 3.78 & 3.31 & 3.35 \\
\hline FCC & 3.77 & 3.80 & 4.18 & 4.19 \\
\hline
\end{tabular}

Table 2. Surface energy for selected surfaces estimated using non-relativistic and relativistic DFT ( $\gamma_{\text {Non-rel. and }} \gamma_{\text {Rel. }}$, respectively) and ReaxFF potential $\left(\gamma_{\text {ReaxFF }}\right)$.

\begin{tabular}{|l|l|l|l|}
\hline Surface & $\gamma_{\text {Non-rel. }}\left(\mathrm{Jm}^{-2}\right)$ & $\gamma_{\text {Rel. }}\left(\mathrm{Jm}^{-2}\right)$ & $\gamma_{\text {ReaxFF }}\left(\mathrm{Jm}^{-2}\right)$ \\
\hline$(1 \times 3)$-pr-Au(110) & 0.89 & 0.85 & 1.23 \\
\hline$(1 \times 2)$-mr-Au(110) & 0.88 & 0.84 & 1.23 \\
\hline $\mathrm{Au}(111)$ & 0.75 & 0.72 & 1.02 \\
\hline$(1 \times 3)$-tr-Au(110) & 0.87 & 0.84 & 1.23 \\
\hline$(5 \times 1)-\mathrm{ar}-\mathrm{Au}(100)$ & 0.87 & 0.84 & 1.36 \\
\hline
\end{tabular}


Fig 1. Oxygen binding positions for (a) (1×3)-pr-Au(110), (b) $(1 \times 2)-m r-A u(110)$ and (c) unreconstructed $\mathrm{Au}(111)$ surfaces. 'mr' and 'pr' denote missing row and pairing row reconstruction, respectively. For each surface the large white, black and light grey circles denote surface, second and third layer Au atoms, respectively, whereas the small, dark grey annotated circles denote oxygen binding positions. In (a) and (b) $(2 \times 3)$ and (2×2) surface supercells are delineated respectively, whereas in (c) a $(3 \times 3)$ supercell is shown; supercells larger than the clean surface reconstruction are delineated for clarity. In (c) the nomenclature ' $\mathrm{H}$ ', 'F', 'A' and ' 2 ' denote the HCP, FCC, atop and two-fold bridge binding positions, respectively, and the subscripts distinguish between multiple binding sites of the same type.

(a)

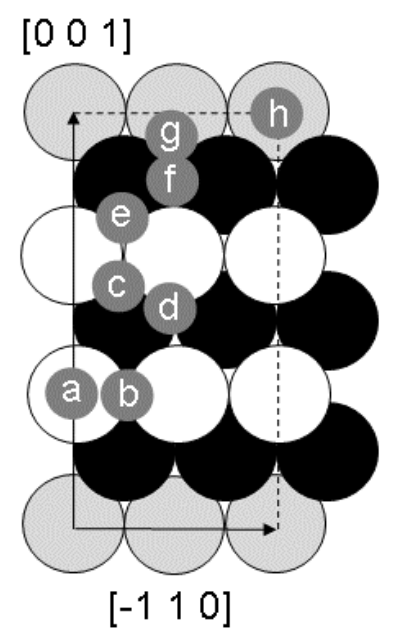


(b)

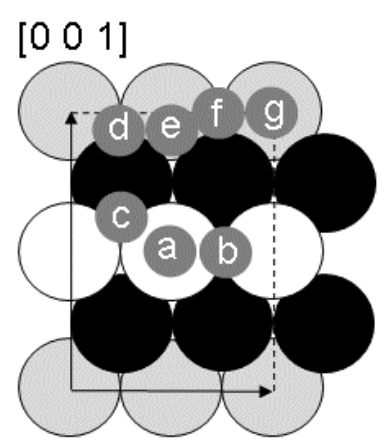

$\left[\begin{array}{lll}-1 & 1 & 0\end{array}\right]$

(c)

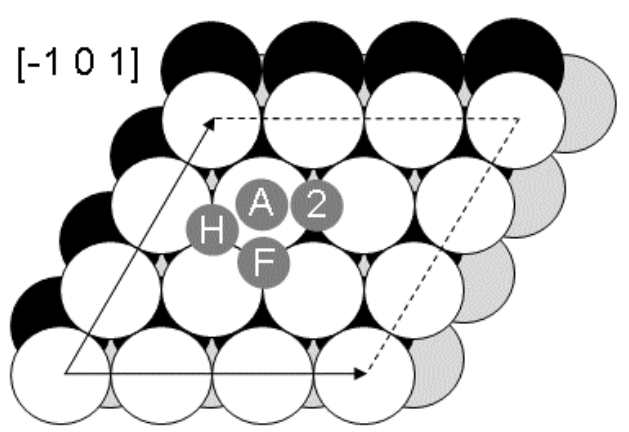

[ $\left.\begin{array}{lll}-1 & 1 & 0\end{array}\right]$ 
Fig. 2. Equations of state for bulk Au arranged in (a) diamond (dia), (b) simple cubic (sc), (c) body-centred cubic (BCC), and (d) face-centred cubic (FCC) structures. Each panel shows the curves for both non-relativistic and relativistic DFT ('Non-rel.' and 'Rel.', respectively) and for the trained ReaxFF potential ('ReaxFF').
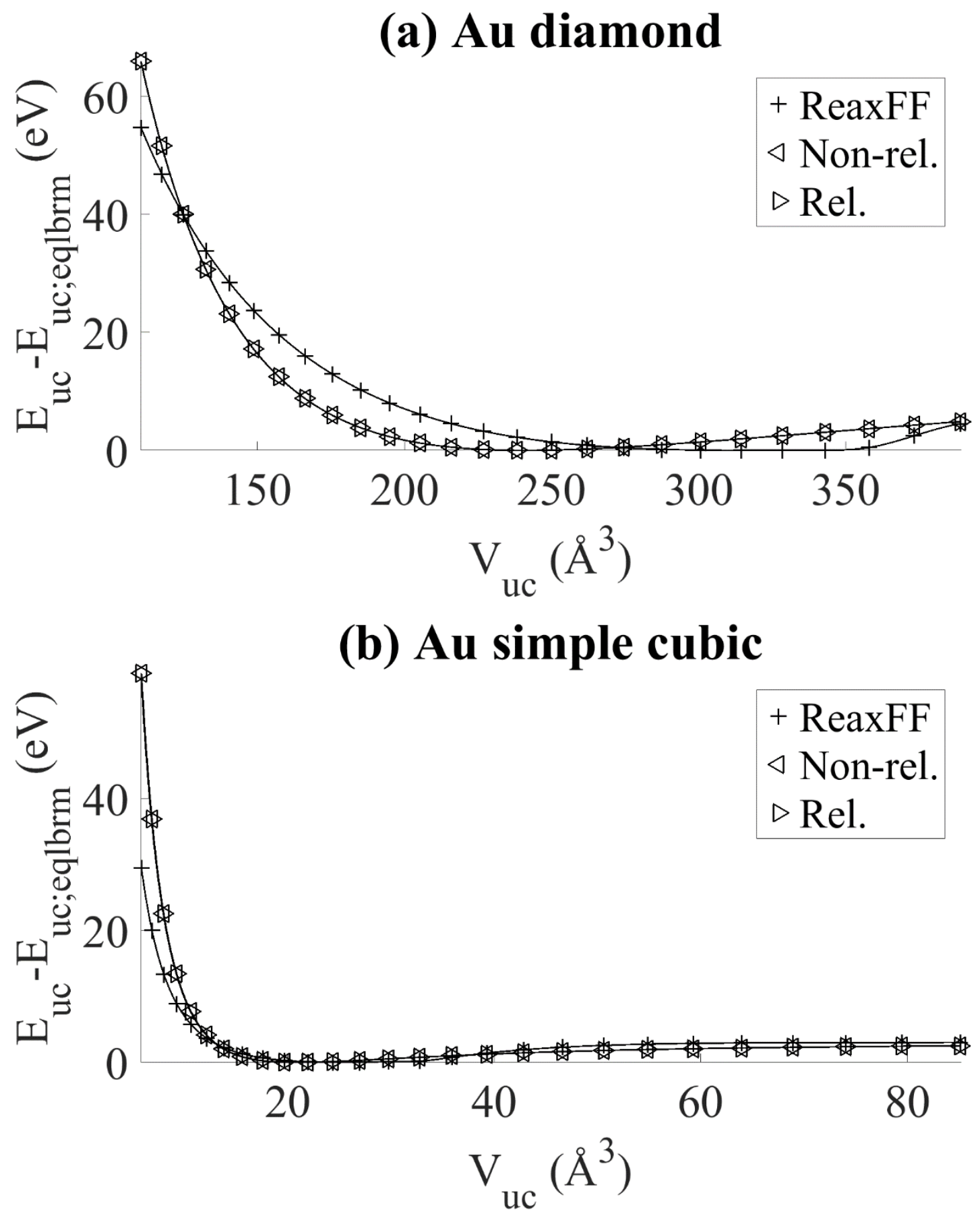

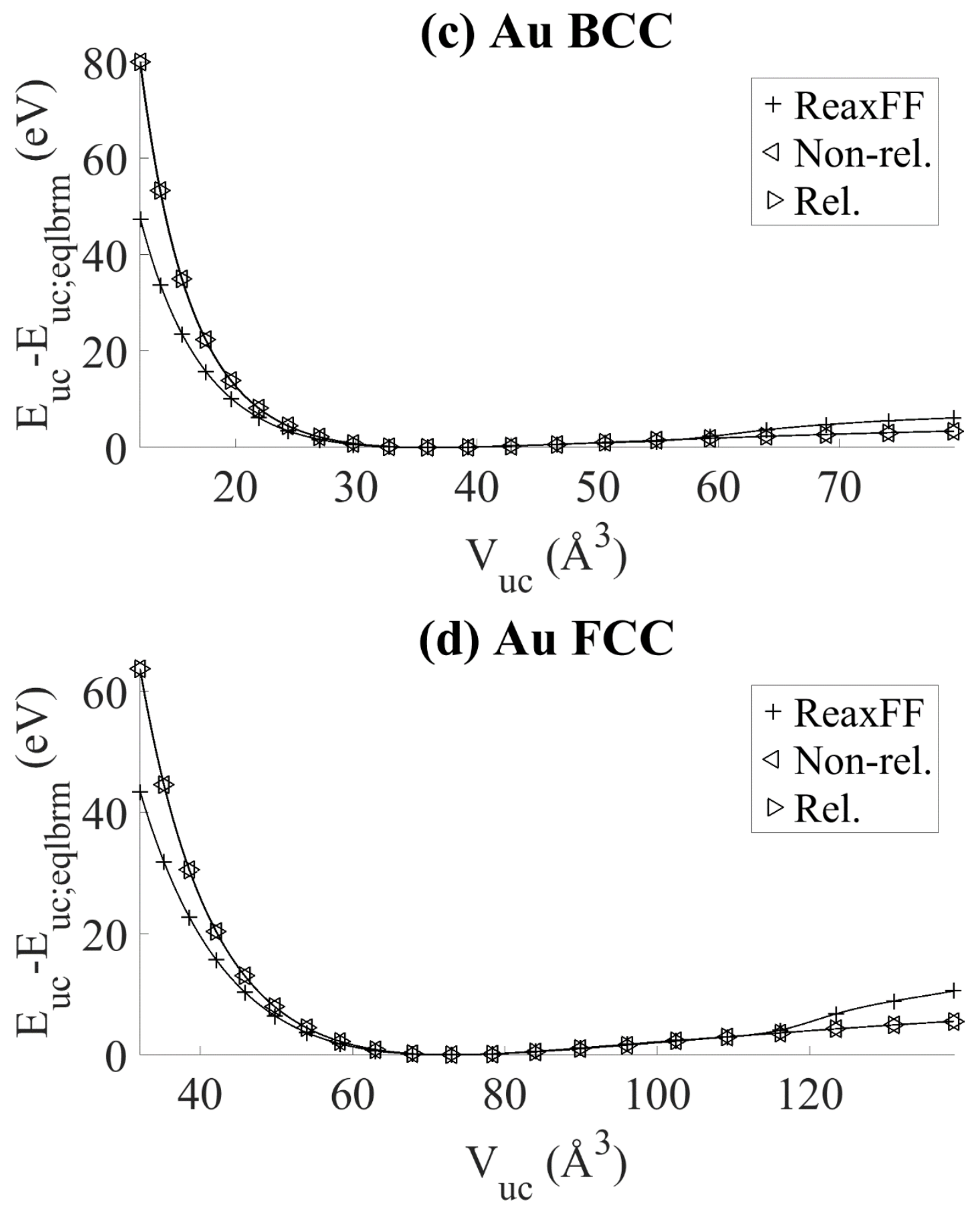
Fig. 3. Equations of state for bulk (a) $\mathrm{Au}_{2} \mathrm{O}_{3}$, and (b) $\mathrm{Au}_{2} \mathrm{O}$ oxides. Each panel shows the curves for both non-relativistic DFT ('Non-rel.') and for the trained ReaxFF potential ('ReaxFF'). 

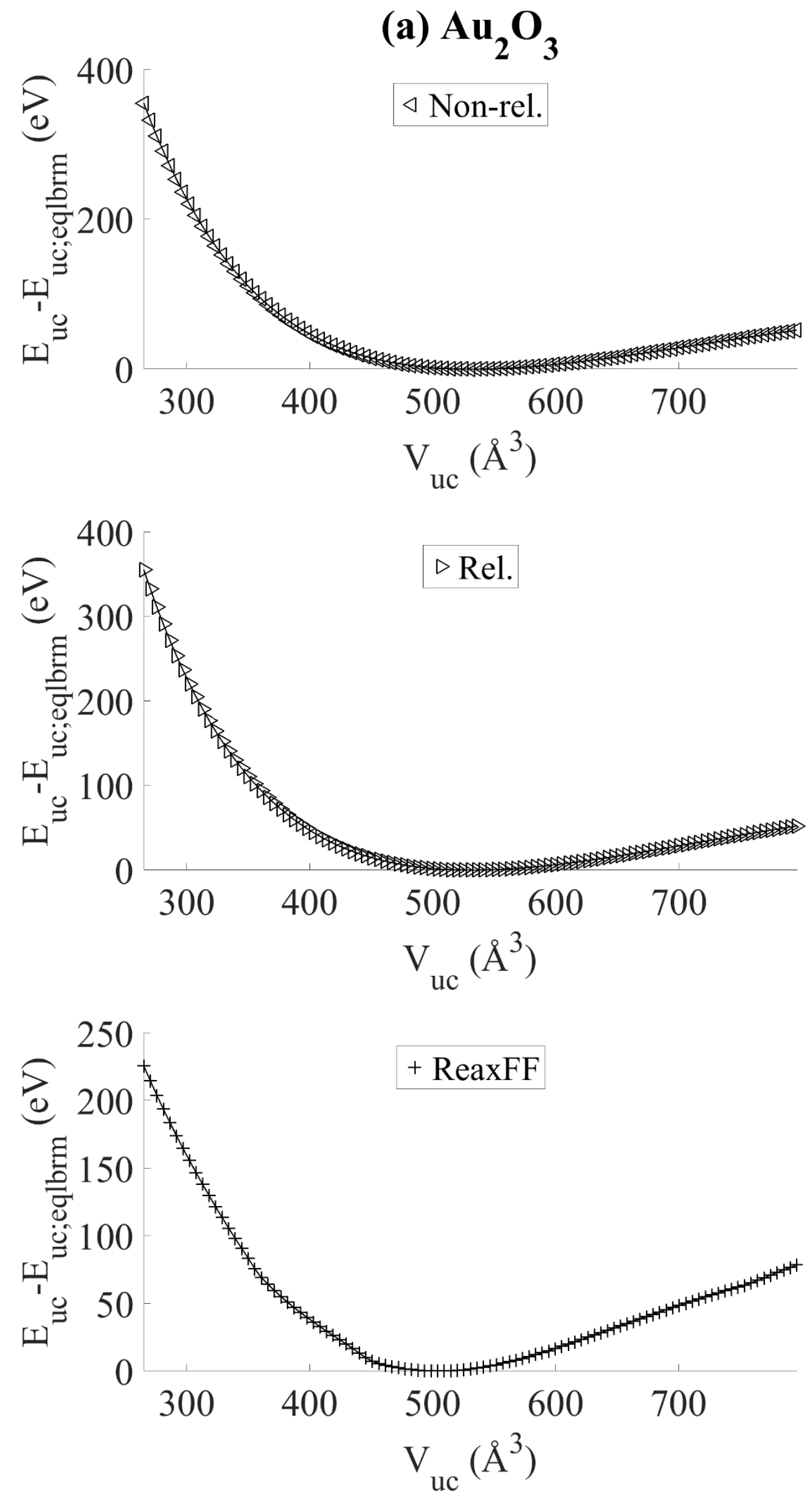
(b) $\mathrm{Au}_{2} \mathrm{O}$
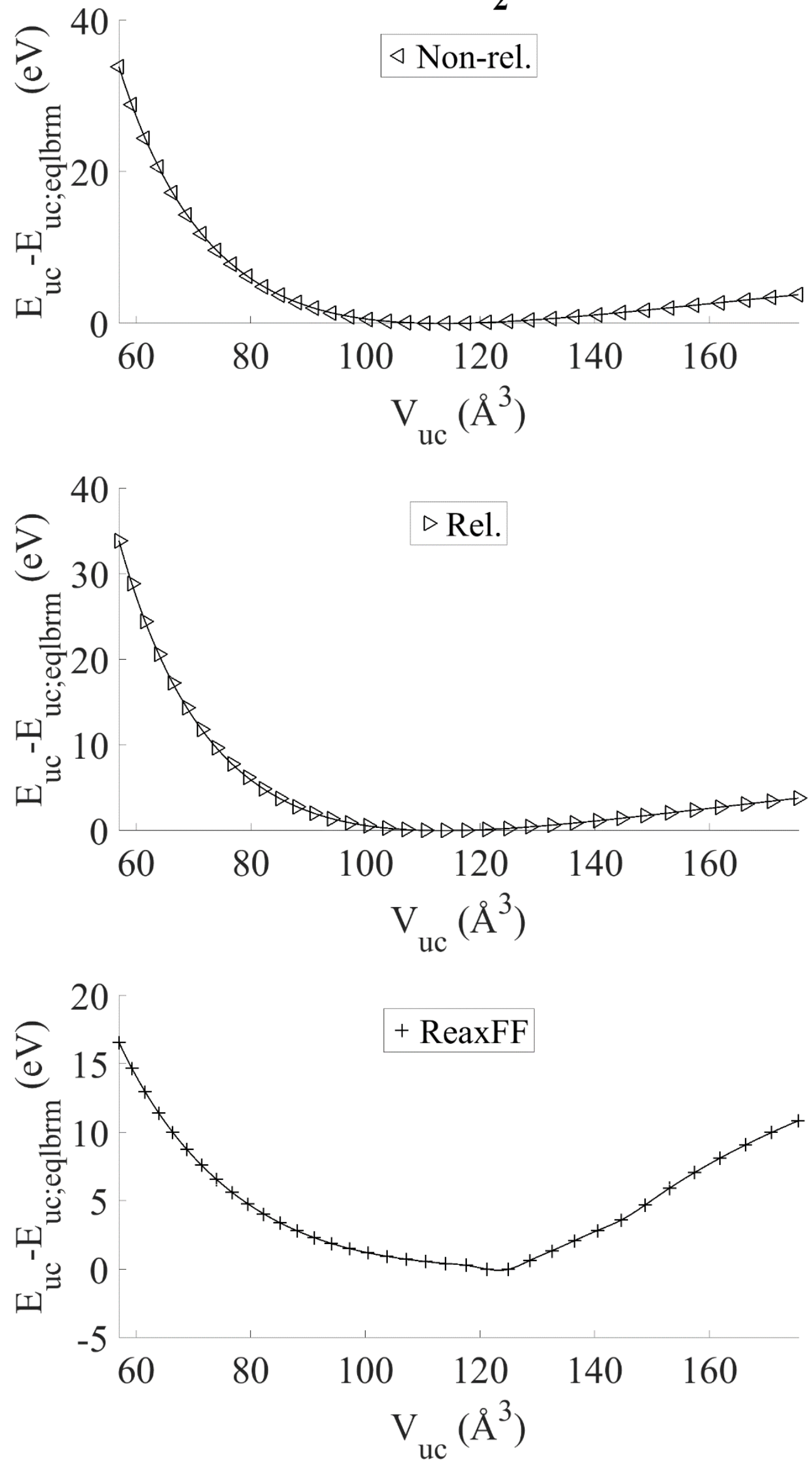
Fig. 4. Equations of state for gas phase $\mathrm{O}_{2}$ obtained using non-relativistic DFT ('Non-rel.') and for the trained ReaxFF potential ('ReaxFF').

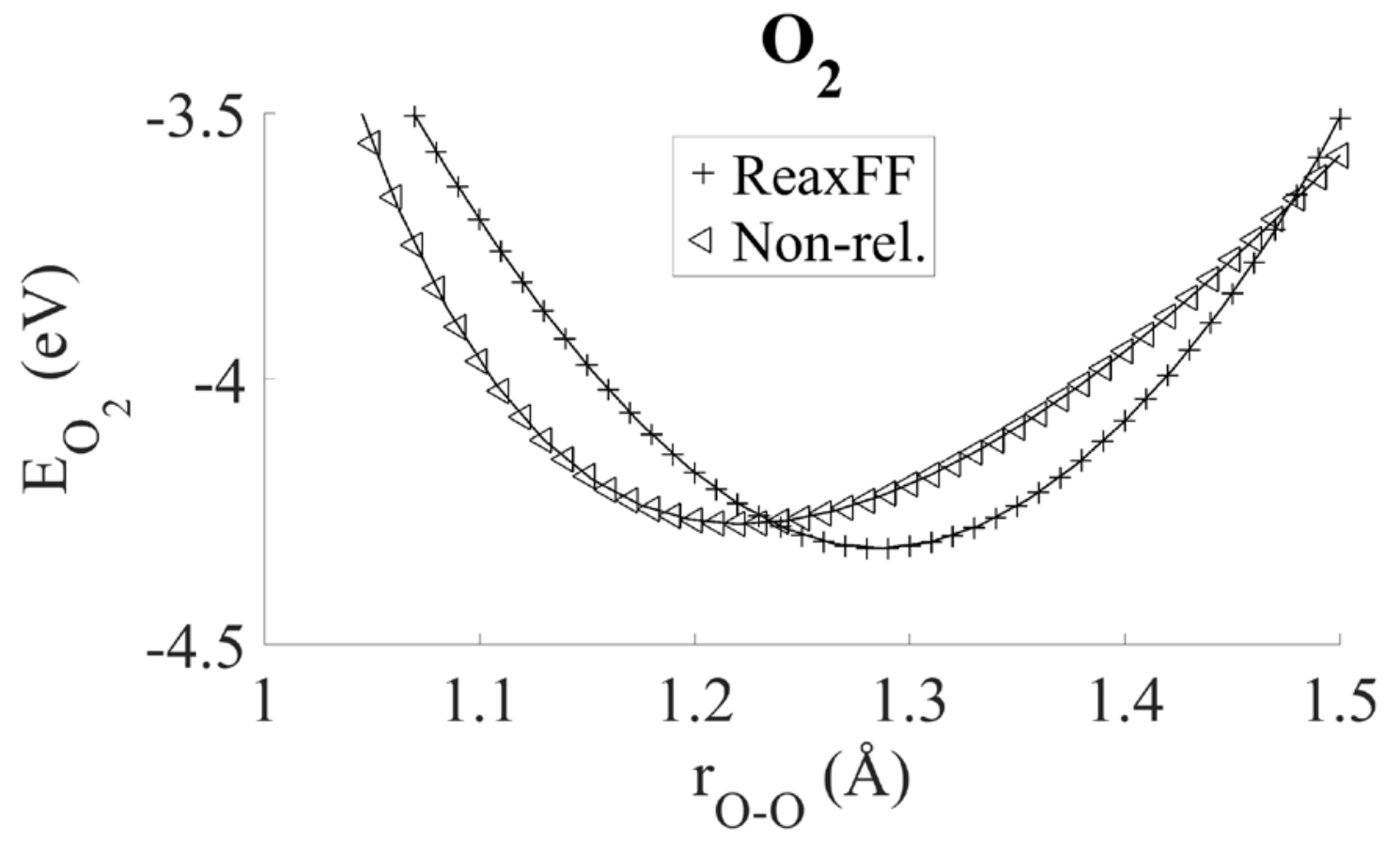


Fig. 5. Isolated oxygen atom binding energies for the (a) $\mathrm{O} /(1 \times 3)-\mathrm{pr}-\mathrm{Au}(110)$, (b) $\mathrm{O} /(1 \times 2)-\mathrm{mr}-$ $\mathrm{Au}(110)$, and (c) $\mathrm{O} /(3 \times 3)-\mathrm{Au}(111)$ surfaces. The oxygen coverage was 1 oxygen atom per surface unit cell, or $0.16,0.25$ or $0.11 \mathrm{ML}$, respectively. Each panel shows the binding energies E for both non-relativistic and relativistic DFT ('Non-rel.' and 'Rel.', respectively) and for the trained ReaxFF potential ('ReaxFF'). The abscissa labels correspond to the binding positions shown in fig. 1.

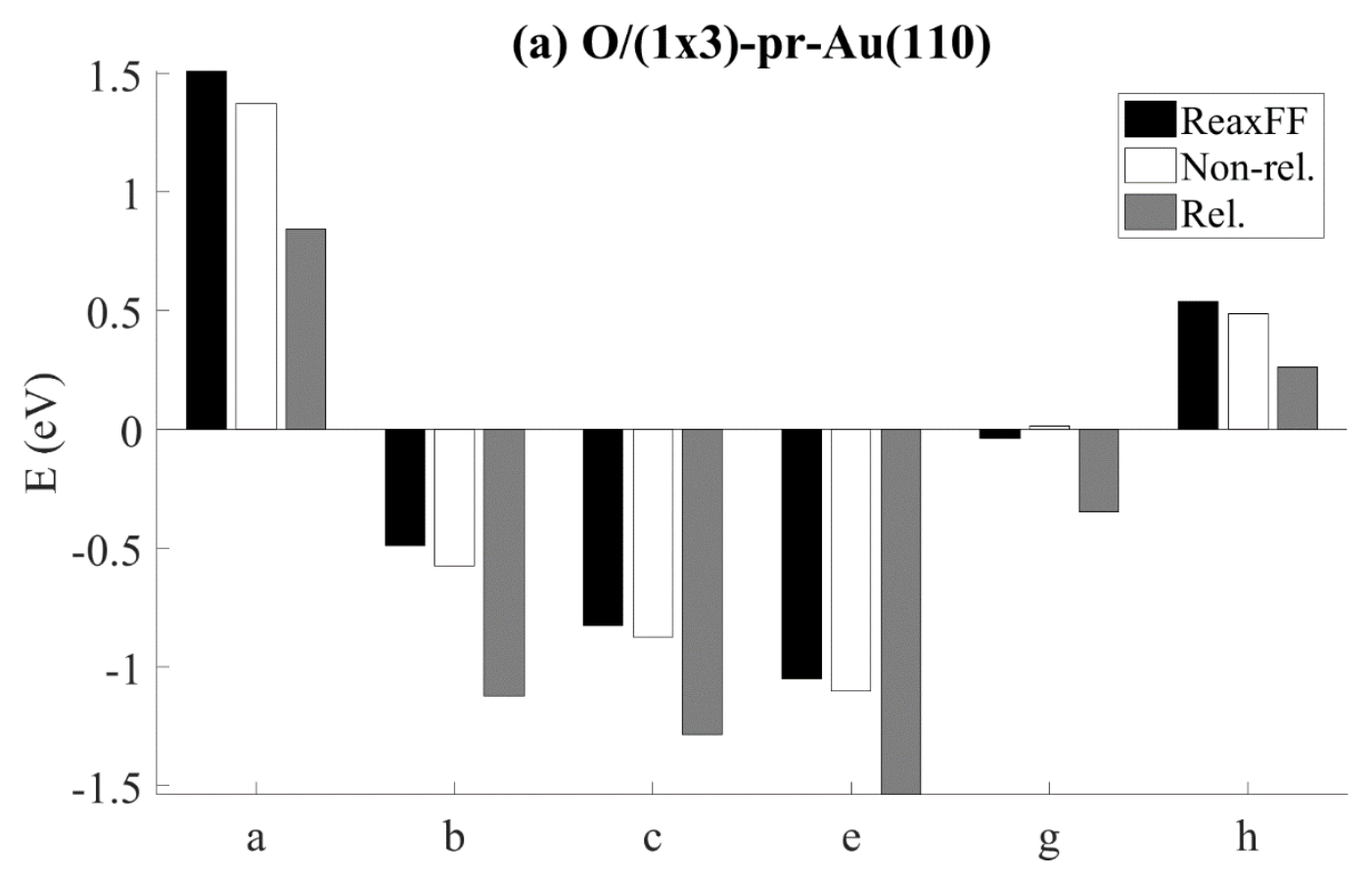


(b) $\mathrm{O} /(1 \times 2)-\mathrm{mr}-\mathrm{Au}(110)$

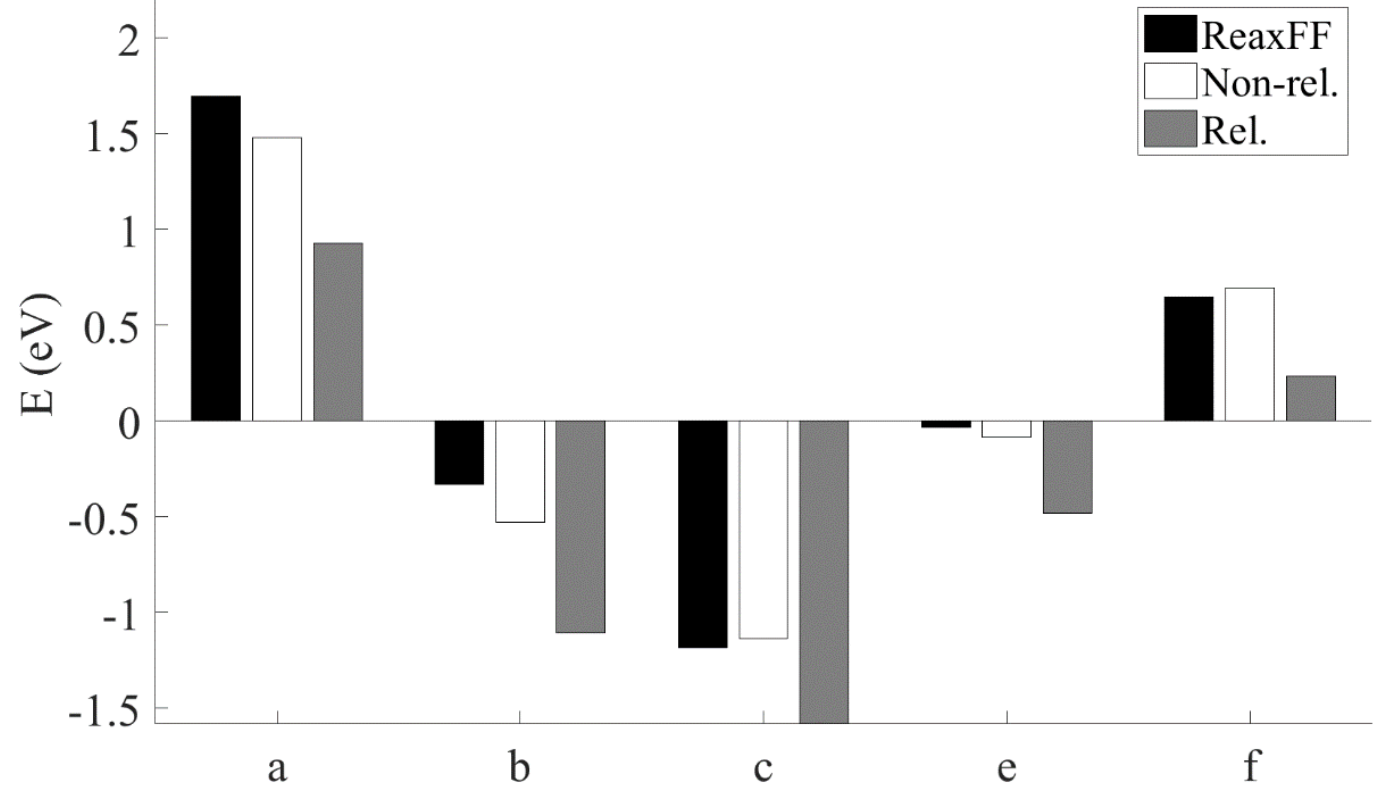




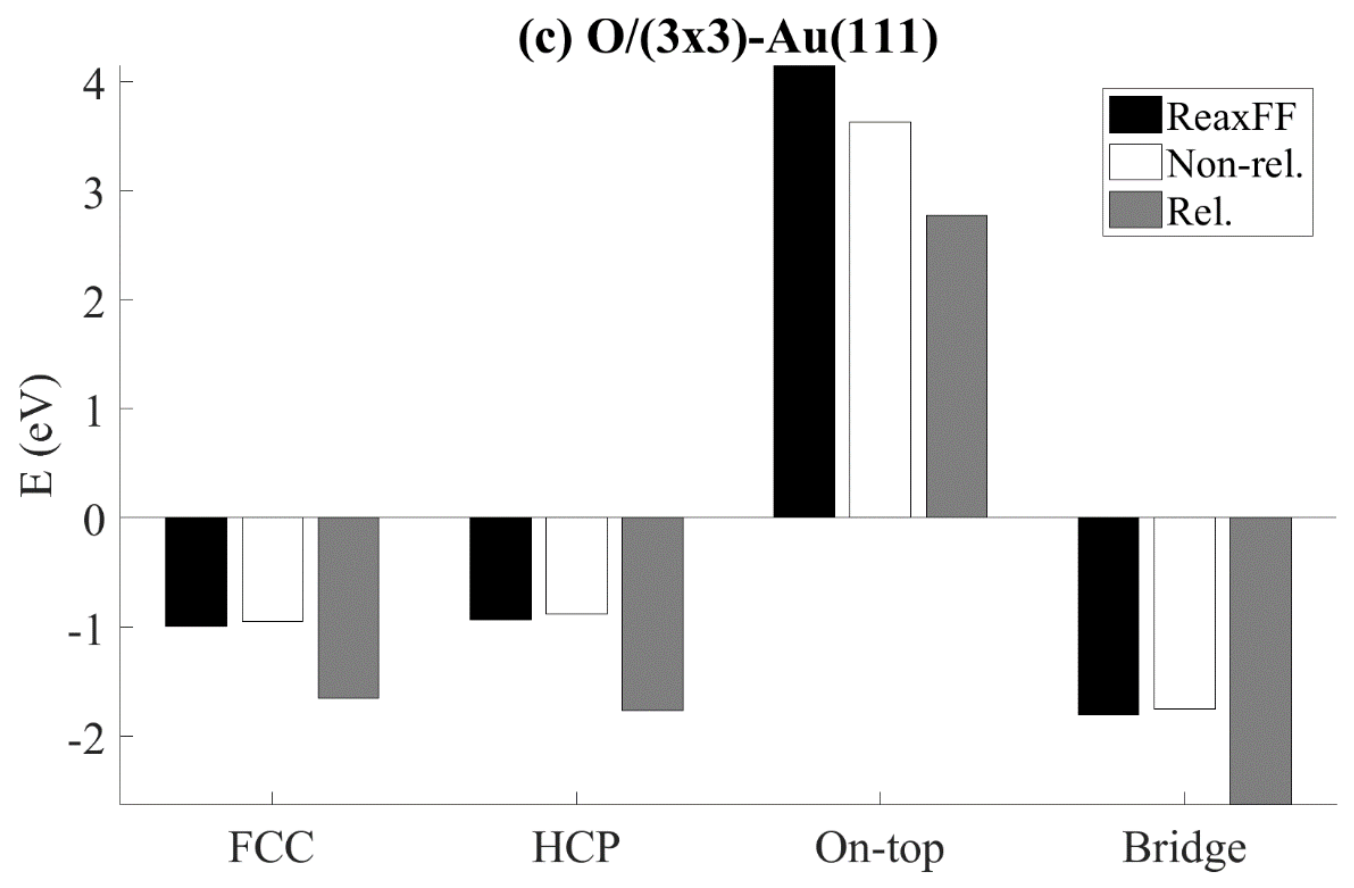


Fig. 6. Oxygen binding energies for the (a) $\mathrm{O}+\mathrm{O} /(1 \times 3)-\mathrm{pr}-\mathrm{Au}(110)$, and (b) $\mathrm{O}+\mathrm{O} /(1 \times 2)-\mathrm{mr}-$ $\mathrm{Au}(110)$ surfaces. The oxygen coverage was 2 oxygen atoms per surface unit cell, or 0.33 or $0.50 \mathrm{ML}$, respectively. Each panel shows the binding energies E for non-relativistic DFT ('Non-rel.') and for the trained ReaxFF potential ('ReaxFF'). The captions show Au and O atoms as gold and red circles, respectively, and the horizontal arrows are oriented along the [$110]$ direction and aligned with the rows of surface Au atoms.

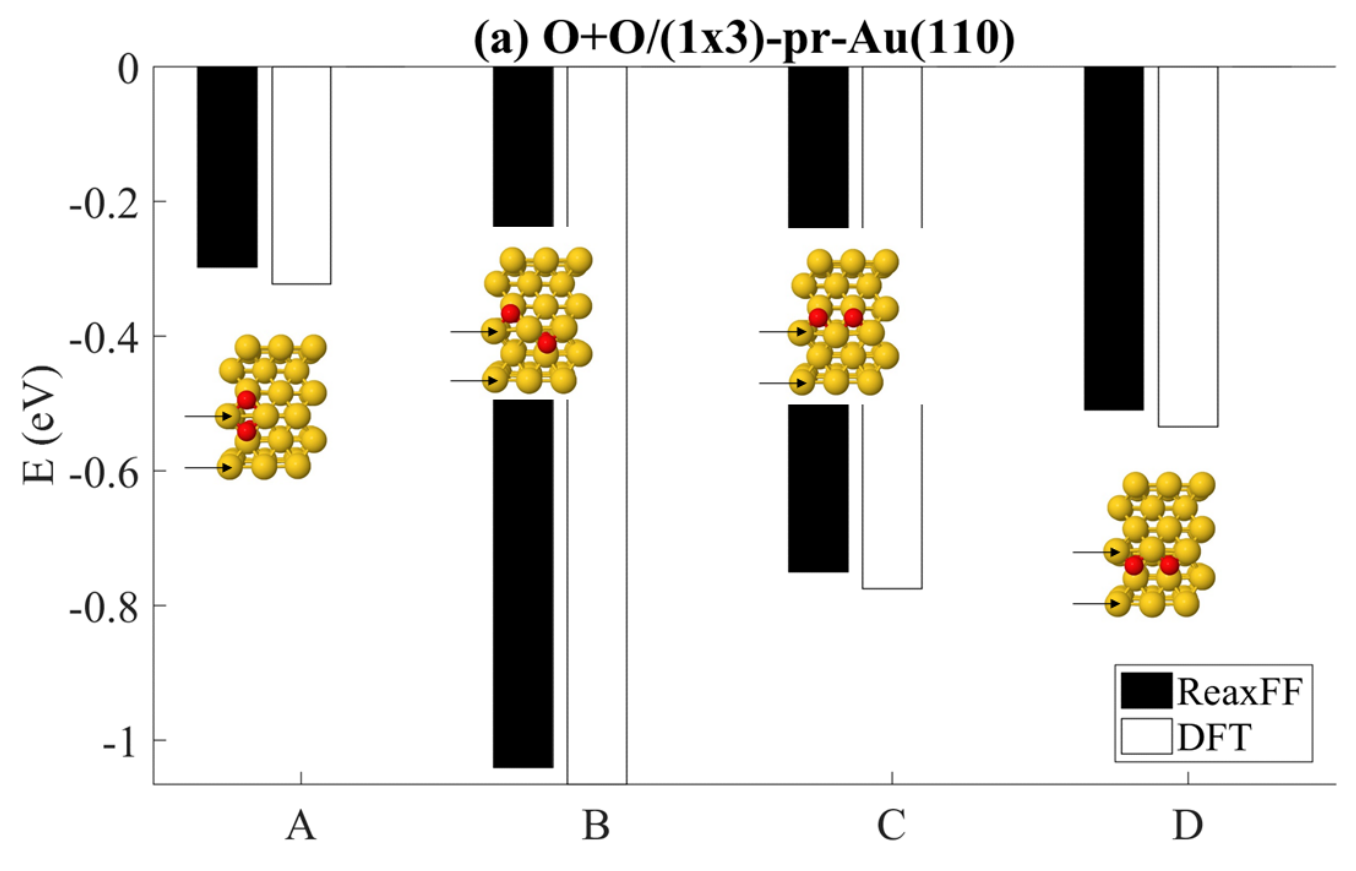


(b) $\mathrm{O}+\mathrm{O} /(\mathbf{1 \times 2})-\mathrm{mr}-\mathrm{Au}(110)$

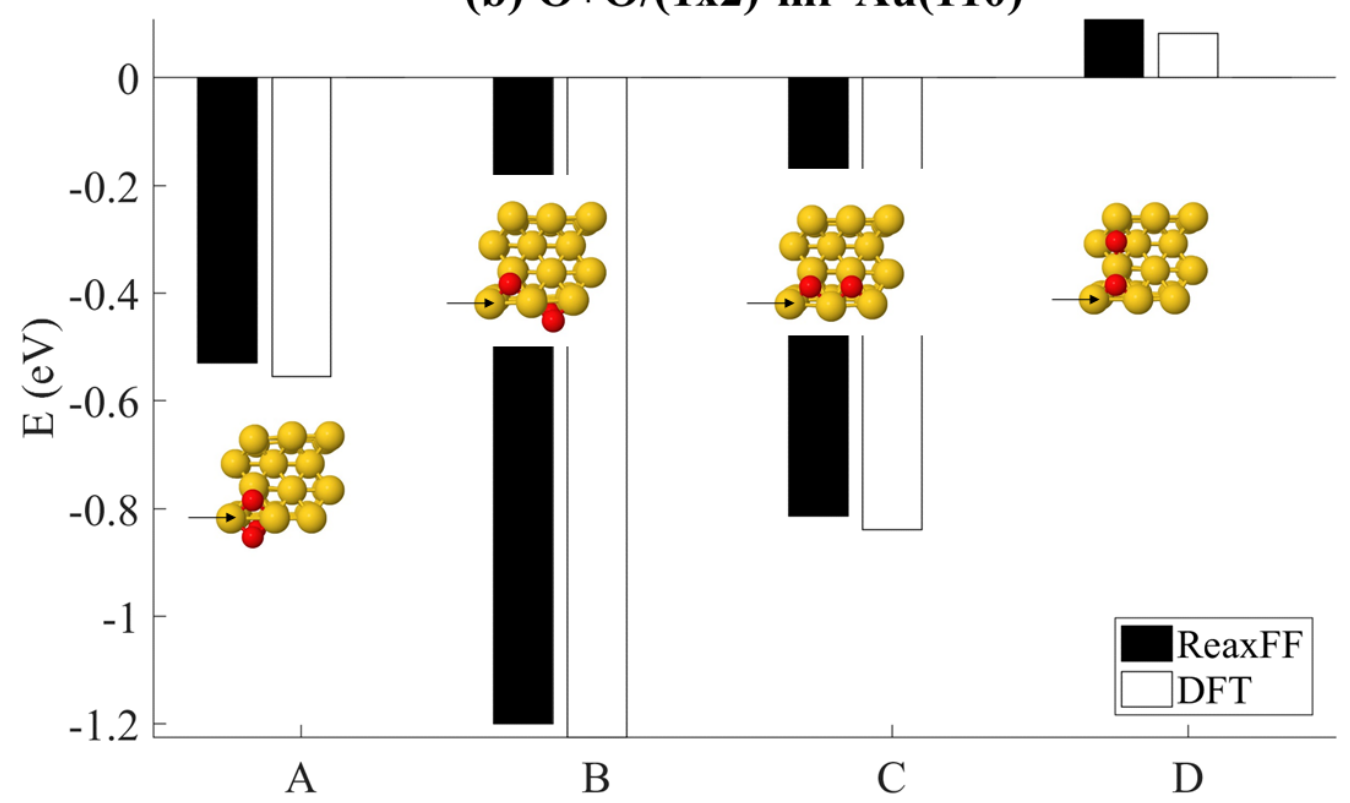


Fig. 7. Nudged elastic band (NEB) pathways across $\mathrm{O}_{2} /(1 \times 3)-\mathrm{pr}-\mathrm{Au}(110)$ with the axis of the $\mathrm{O}_{2}$ aligned initially parallel to the (a) $\left[\begin{array}{lll}0 & 0 & 1\end{array}\right]$, (b) [ $\left[\begin{array}{lll}1 & -1 & 1\end{array}\right]$ and (c-d) $\left[\begin{array}{lll}-1 & 1 & 0\end{array}\right]$ directions. The reaction coordinate is defined with the un-dissociated $\mathrm{O}_{2}$ in the gas phase on the left-most, and with the fully dissociated surface $\mathrm{O}$ atoms rightmost. The captions show $\mathrm{Au}$ and $\mathrm{O}$ atoms as gold and red circles, respectively, and the pairs of horizontal arrows are oriented along the [-1 10 ] direction and aligned with the rows of surface Au atoms. Each group of pathways (i-iii/iv) contain different initial position of the gas phase $\mathrm{O}_{2}$ molecule, and in (c) and (d) the final dissociated $\mathrm{O}$ atoms lie on the trench side of the rows of surface Au atoms and intercalated within the rows of surface Au atoms, respectively. 
(a)(i) $\mathrm{NEB} \mathrm{O}_{2} /(1 \times 3)-p r-A u(110)$
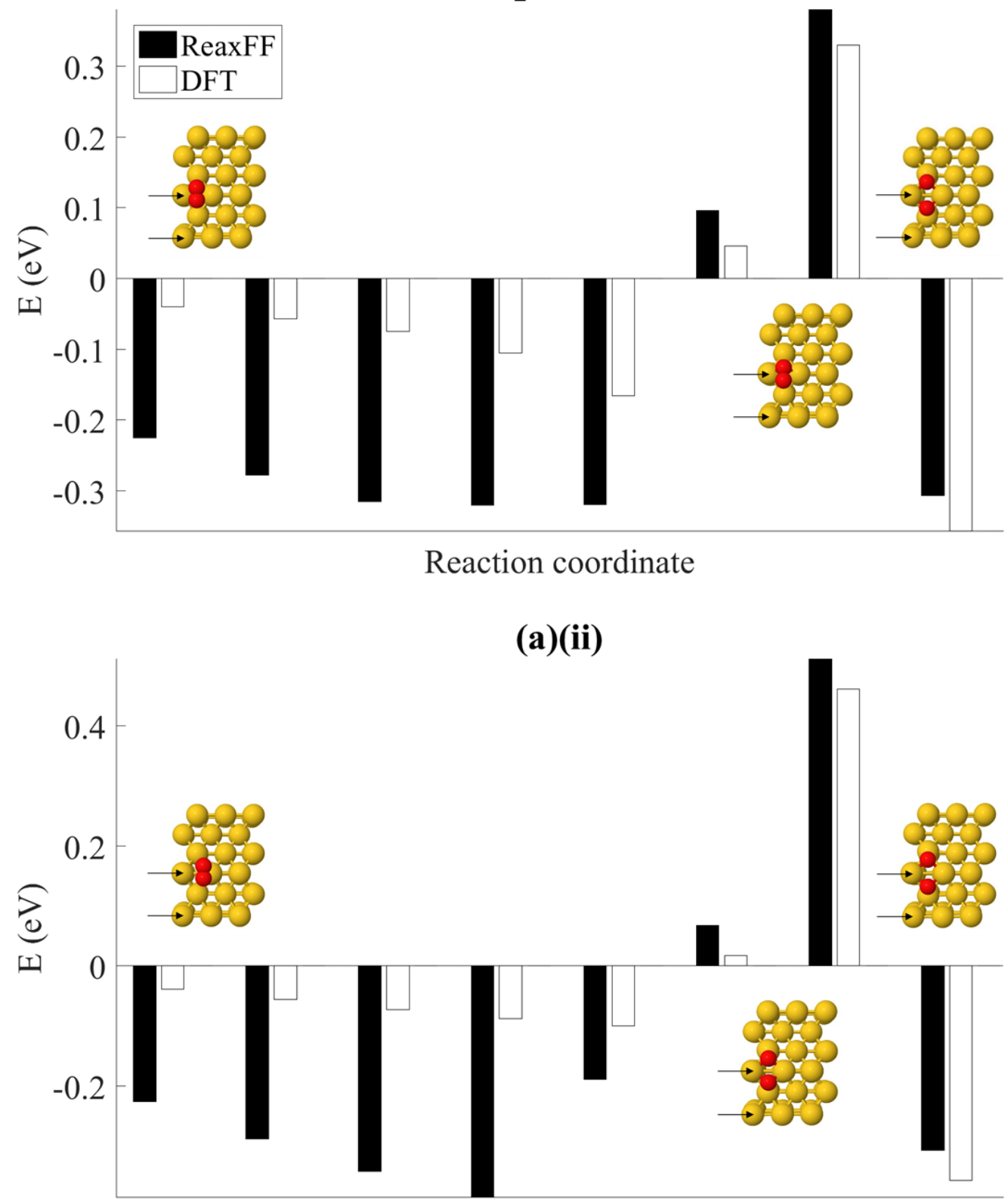

Reaction coordinate 

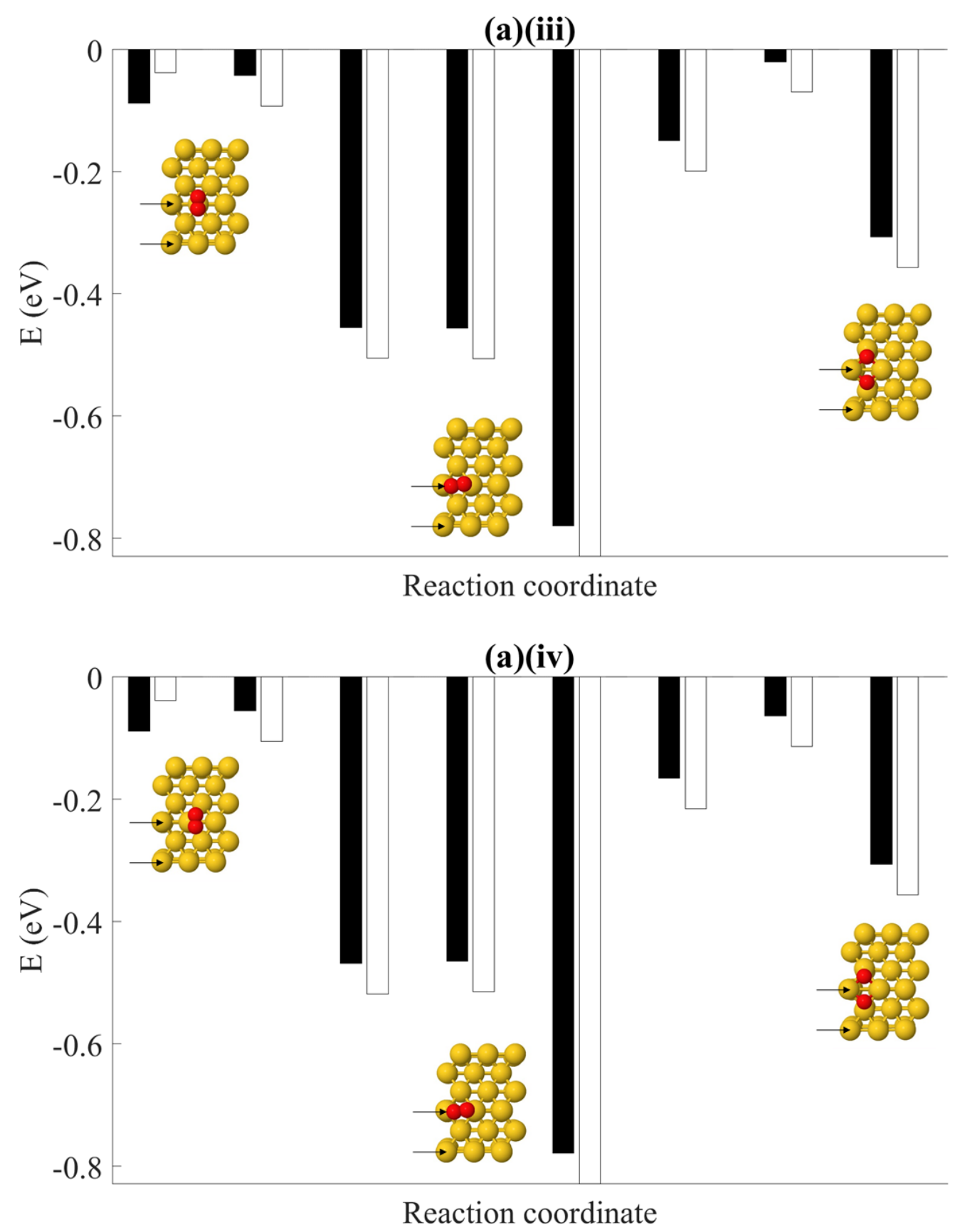

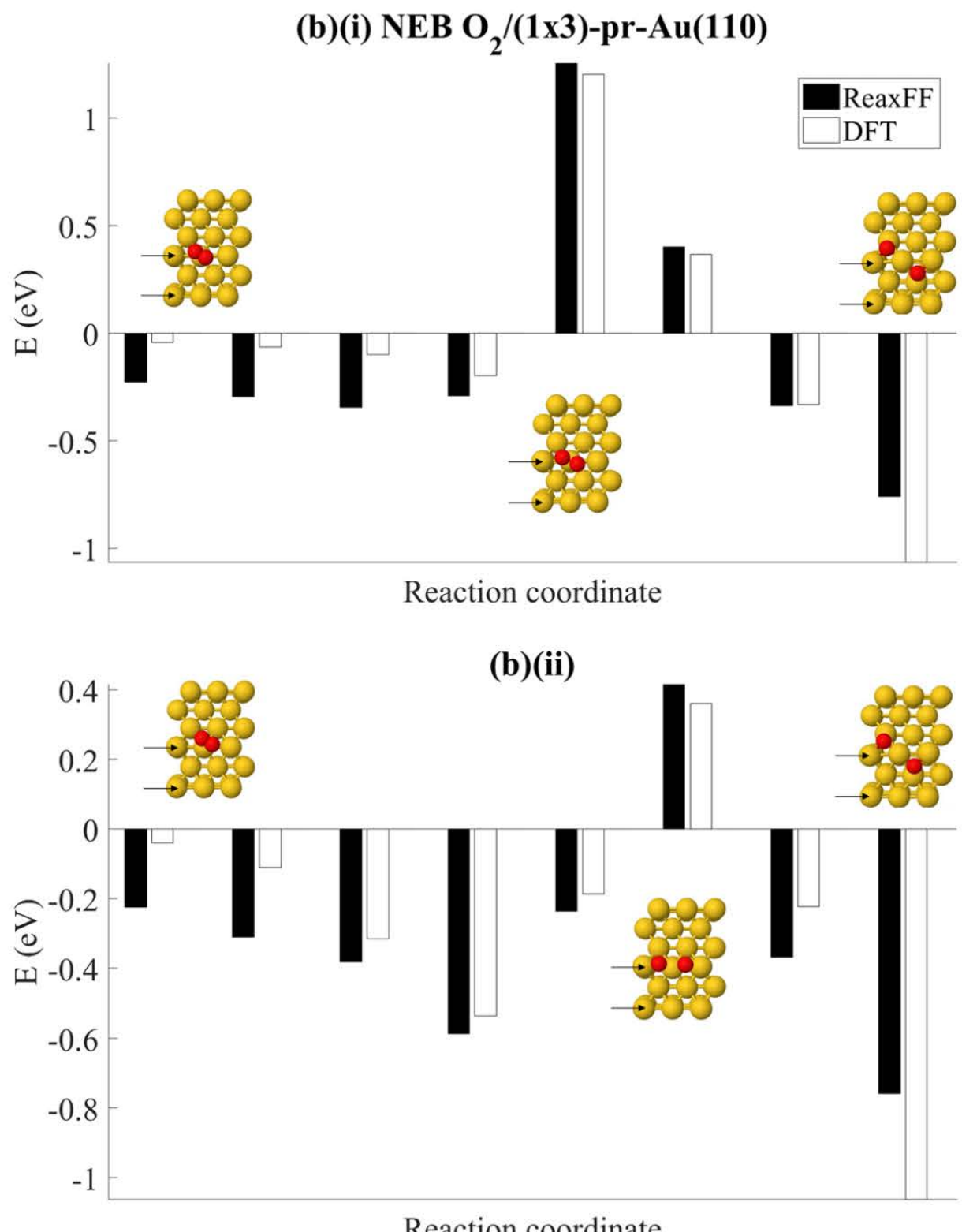

Reaction coordinate

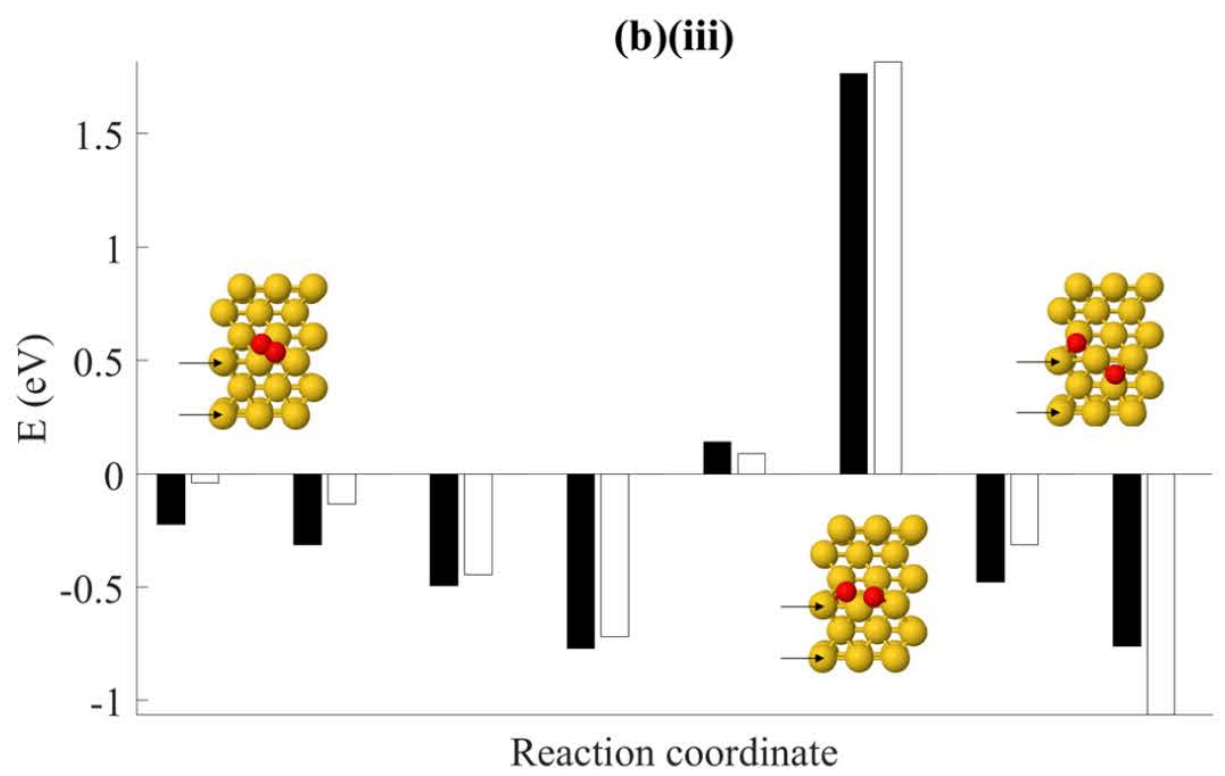




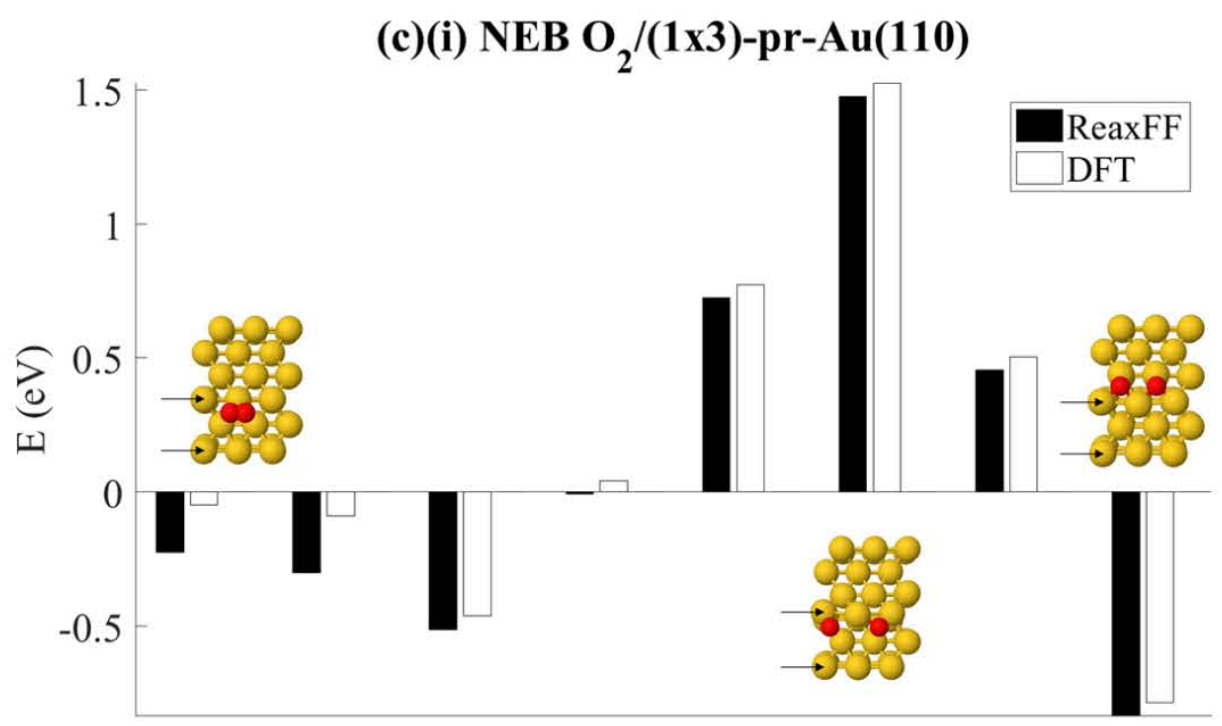

Reaction coordinate

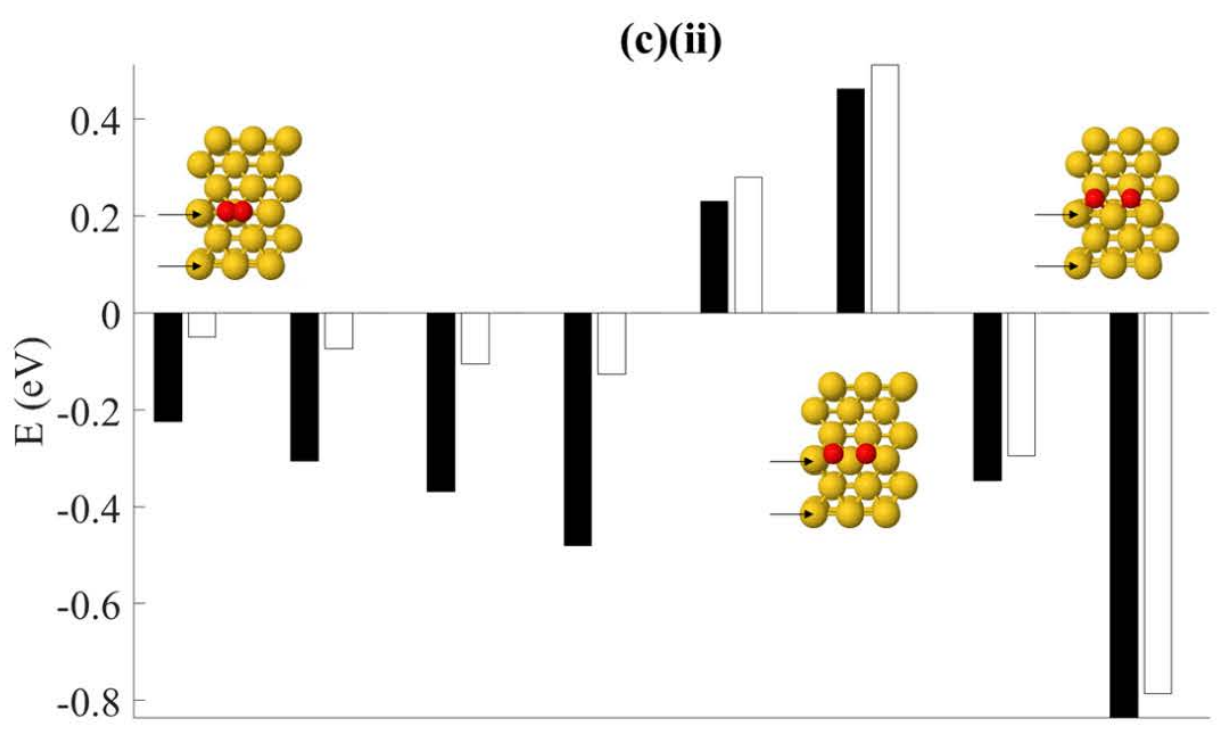

Reaction coordinate

(c)(iii)

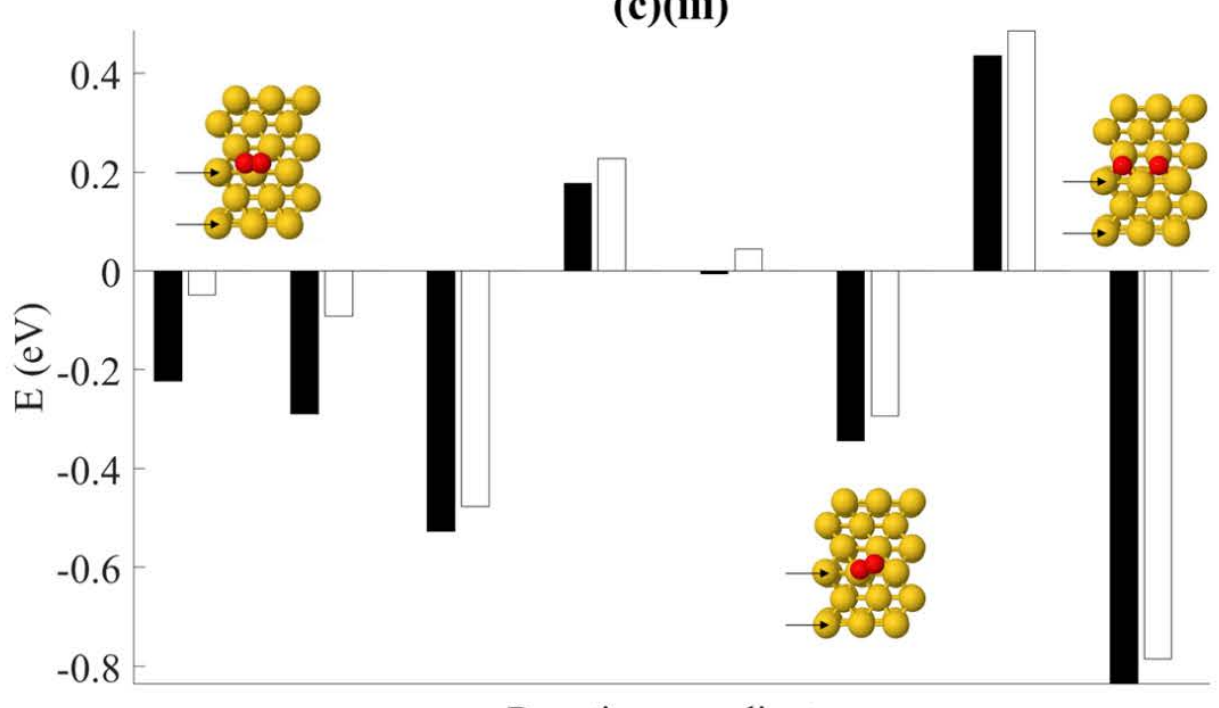

Reaction coordinate 

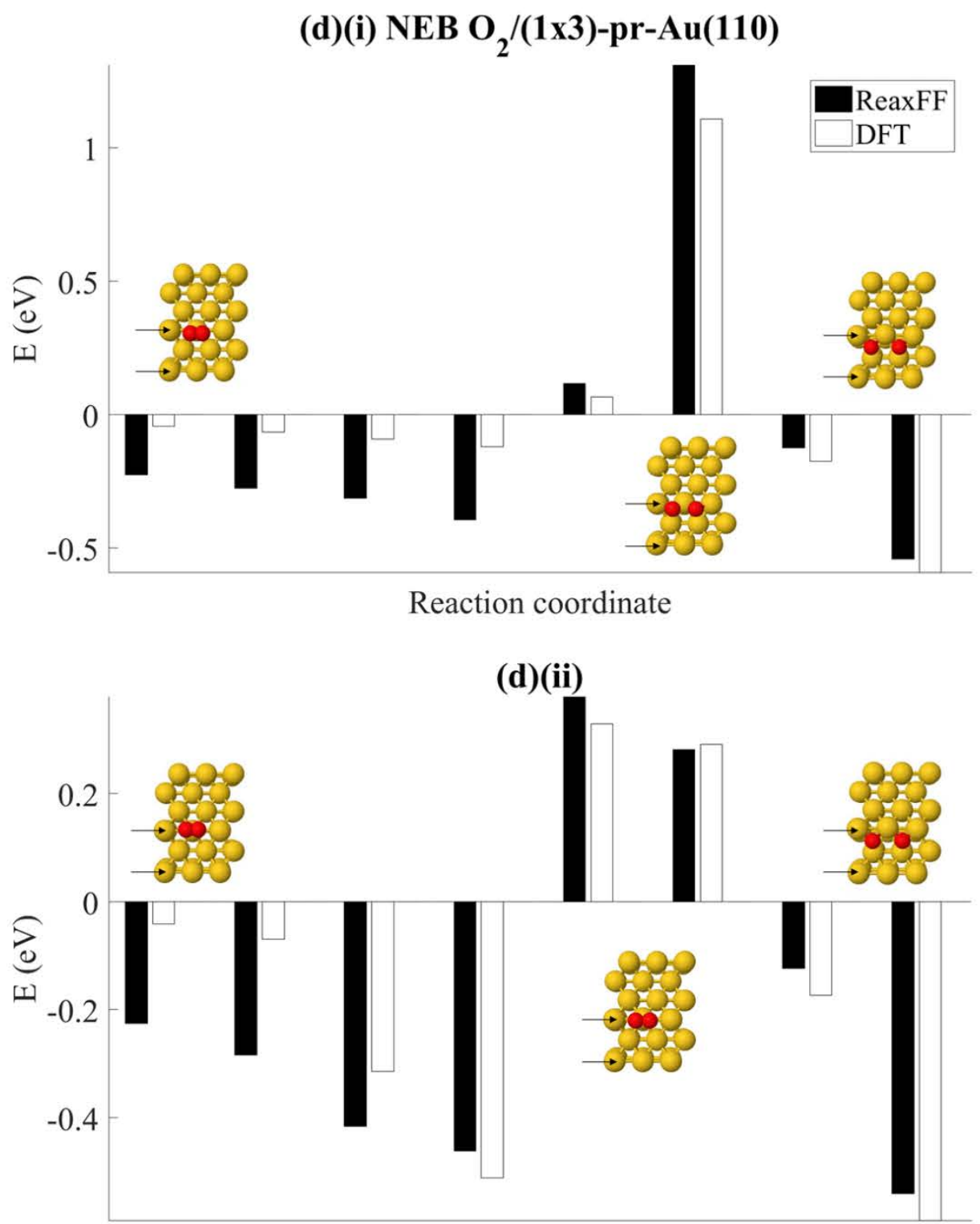

Reaction coordinate

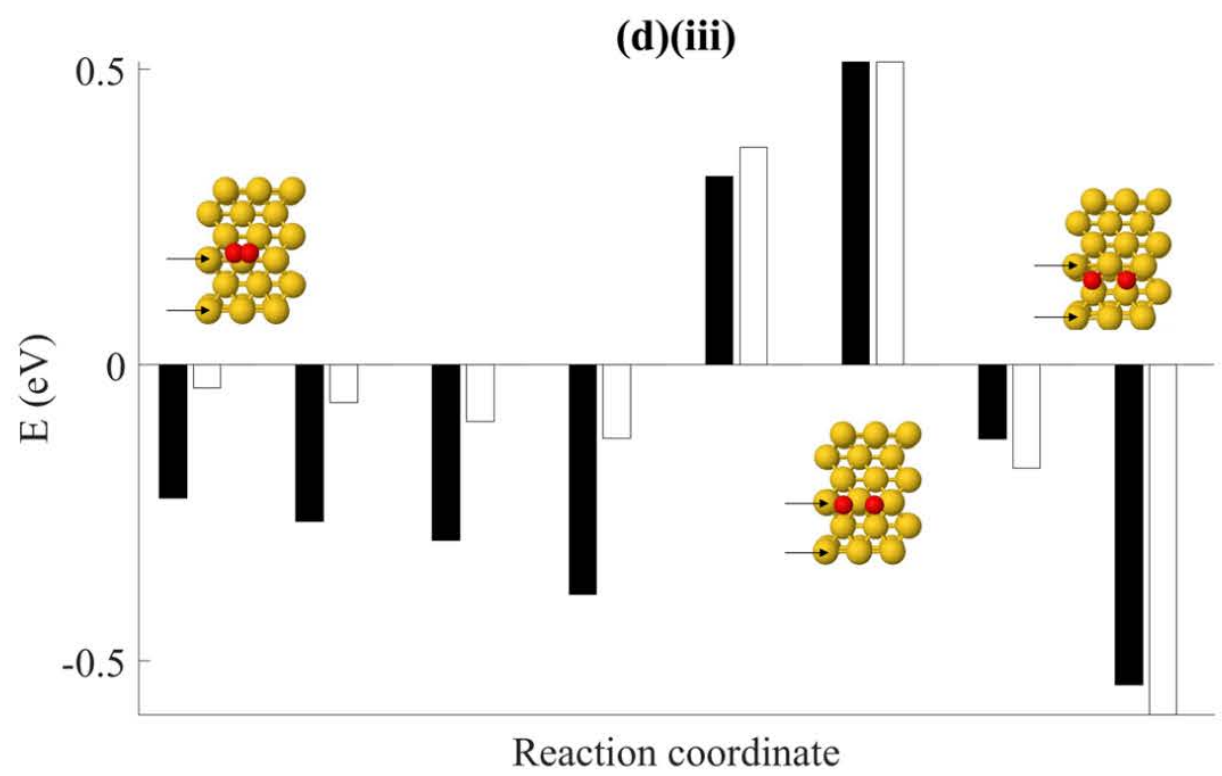


Fig. 8. Nudged elastic band (NEB) pathways across $\mathrm{O}_{2} /(1 \times 2)-m r-\mathrm{Au}(110)$ with the axis of the $\mathrm{O}_{2}$ aligned initially parallel to the [1 -11 1] direction. The reaction coordinate is defined with un-dissociated $\mathrm{O}_{2}$ in the gas phase on the left-most, and with fully dissociated surface $\mathrm{O}$ atoms rightmost. The captions show $\mathrm{Au}$ and $\mathrm{O}$ atoms as gold and red circles, respectively. The horizontal arrows are oriented along the $\left[\begin{array}{lll}-1 & 1 & 0\end{array}\right]$ direction and aligned with the surface $\mathrm{Au}$ atoms. Each pathway (a-b) corresponds to a different initial position of the gas phase $\mathrm{O}_{2}$ molecule, and the fully dissociated $\mathrm{O}$ atoms occupy equivalent positions on either side of the row of surface Au atoms.

(a) $\mathrm{NEB} \mathrm{O}_{2} /(1 \times 2)-\mathrm{mr}-\mathrm{Au}(110)$

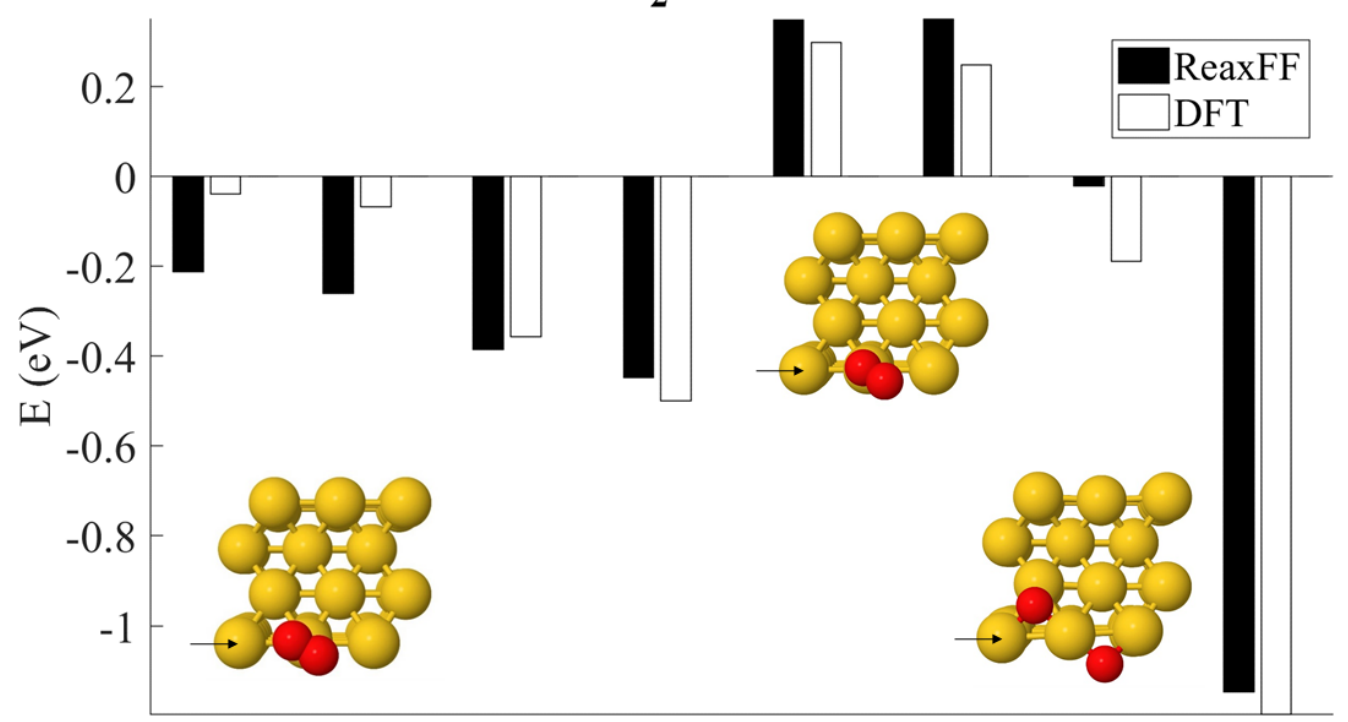

Reaction coordinate

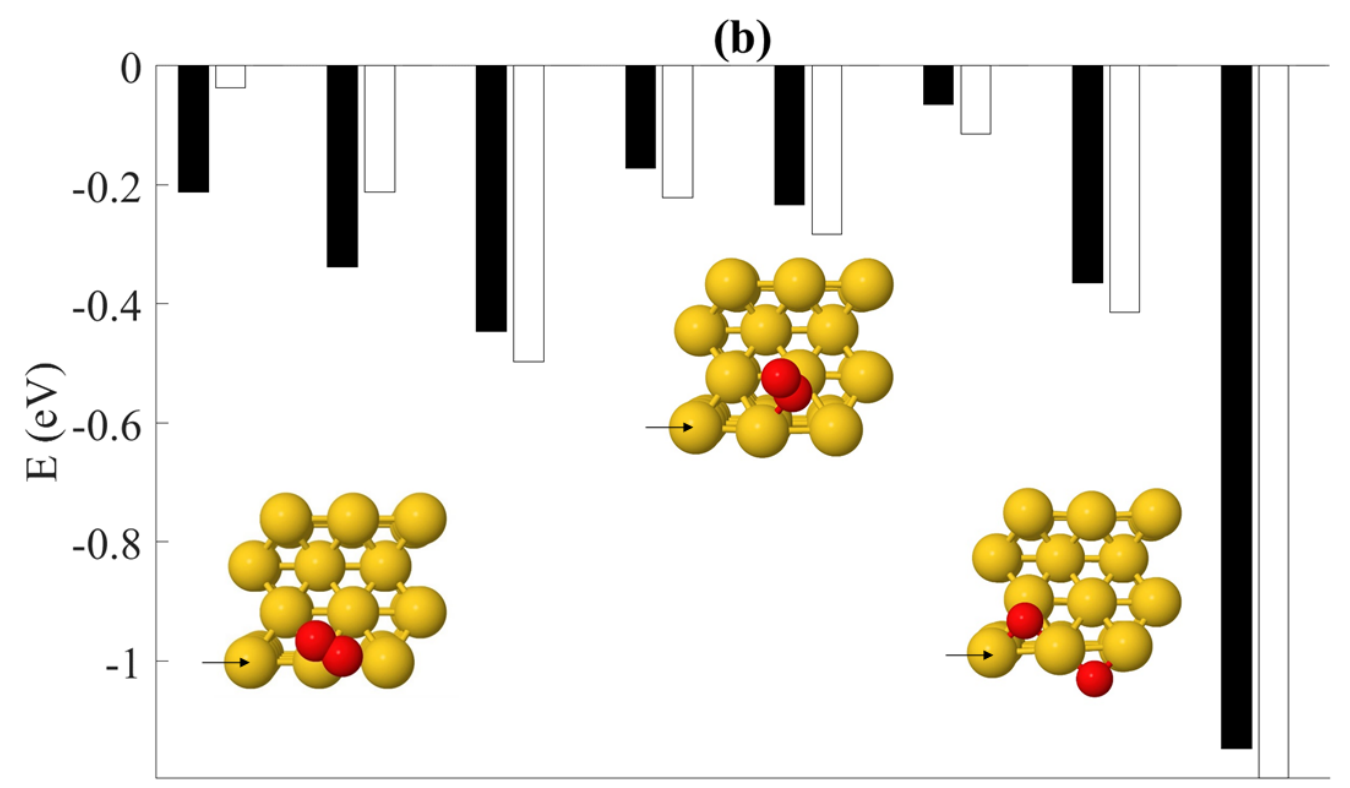

Reaction coordinate 
Fig. 9. Temperature ramped NVT simulations of $\mathrm{O}_{2}$ on the (a) $(1 \times 3)-\mathrm{pr}-\mathrm{Au}(110)$, (b) $(1 \times 2)$ mr-Au(110), (c) $\mathrm{Au}(111)$, (d) (1×3)-tr-Au(110), and (e) (5×1)-ar-Au(100) surfaces.

(a) $(1 \times 3)-p r-A u(110)$
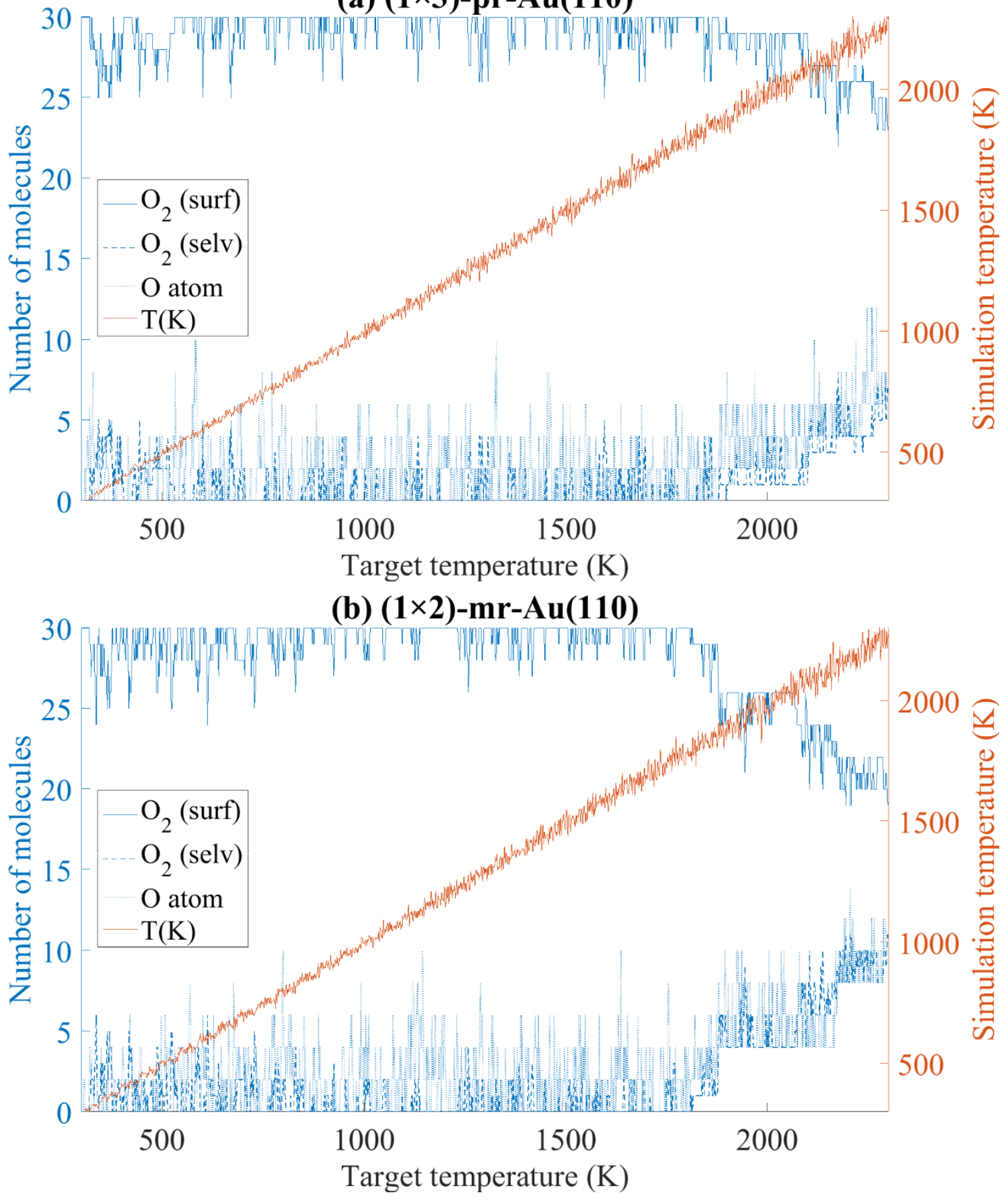

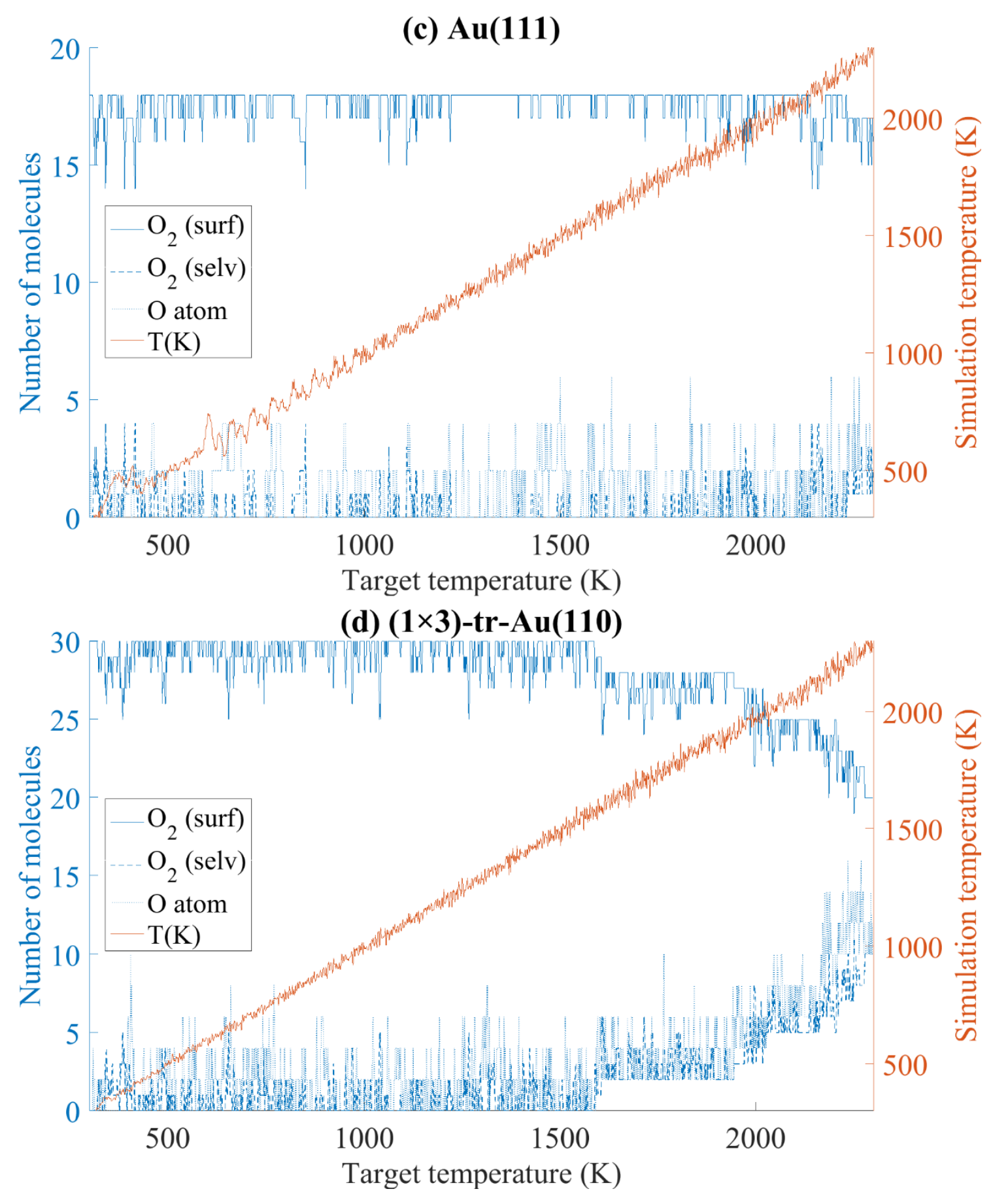


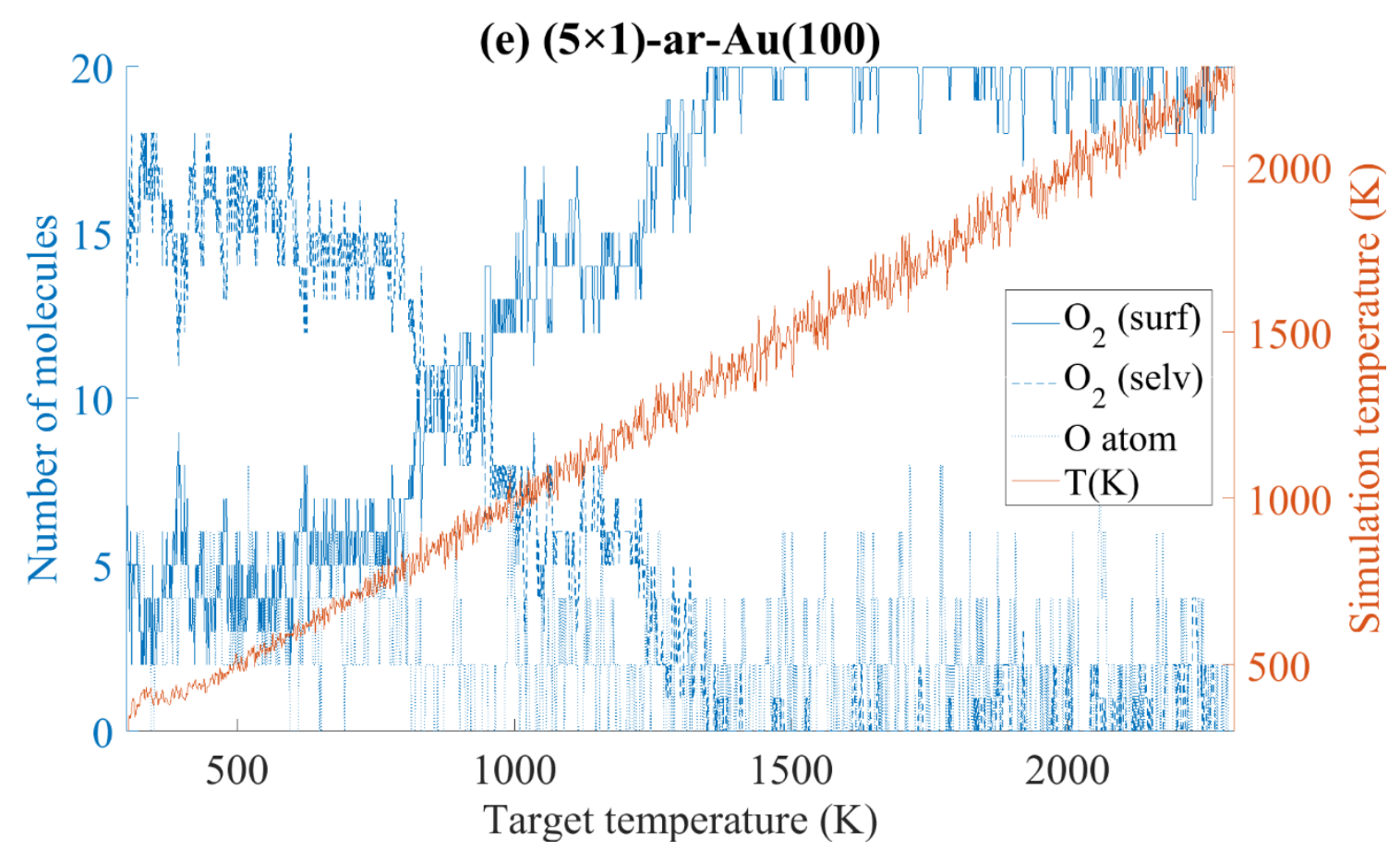


Fig. 10. Temperature ramped NVT simulations of $\mathrm{O}_{2}$ on the (a) $(1 \times 3)-\mathrm{pr}-\mathrm{Au}(110),(\mathrm{b})(1 \times 2)$ mr-Au(110), (c) Au(111), (d) (1×3)-tr-Au(110), and (e) (5×1)-ar-Au(100) surfaces. Each panel shows the laterally averaged Au atom density summed along the [-1 10$]$ direction .The colour scale to the right of each graph shows the laterally averaged number density of Au atoms in units of atoms/unit cell.
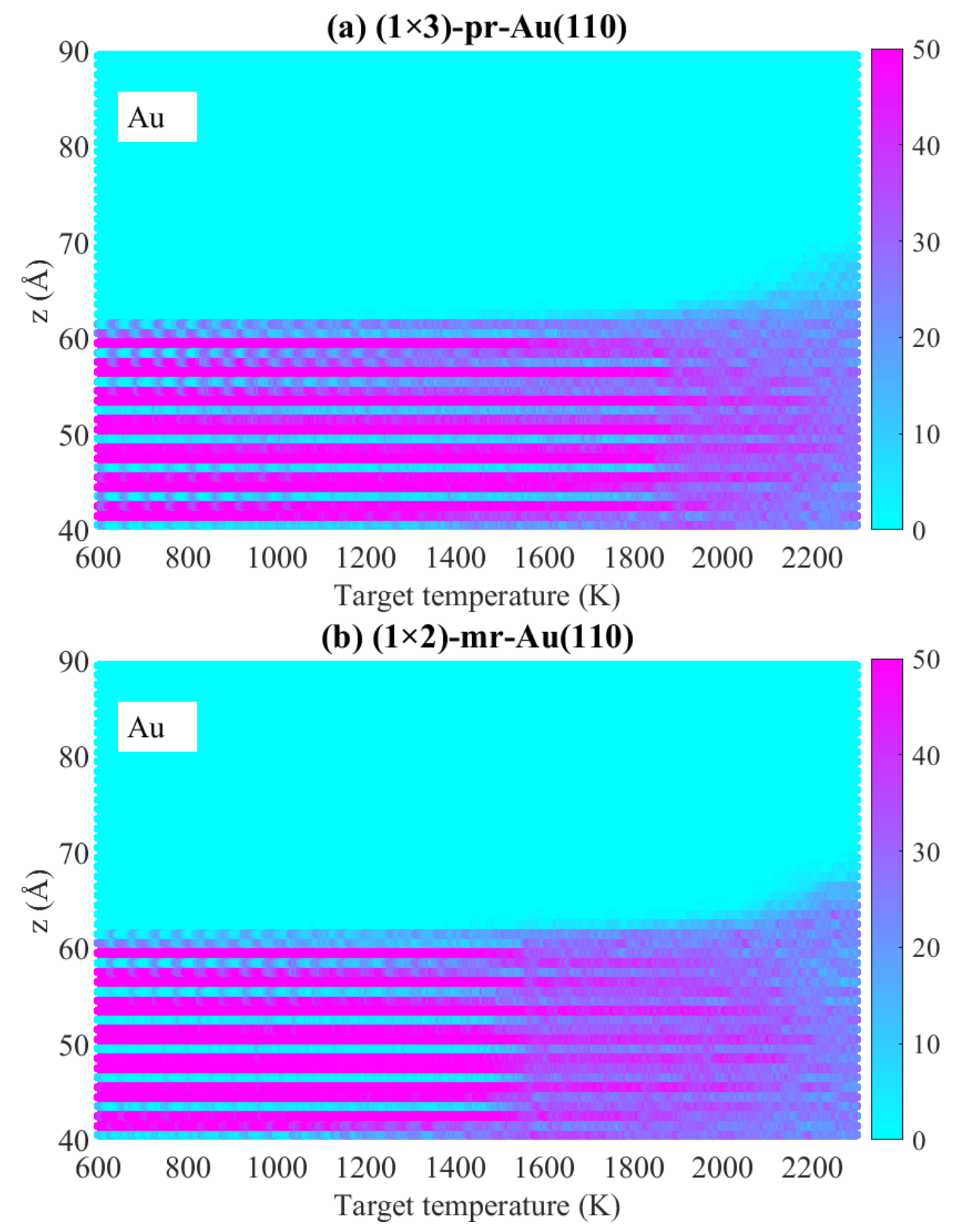

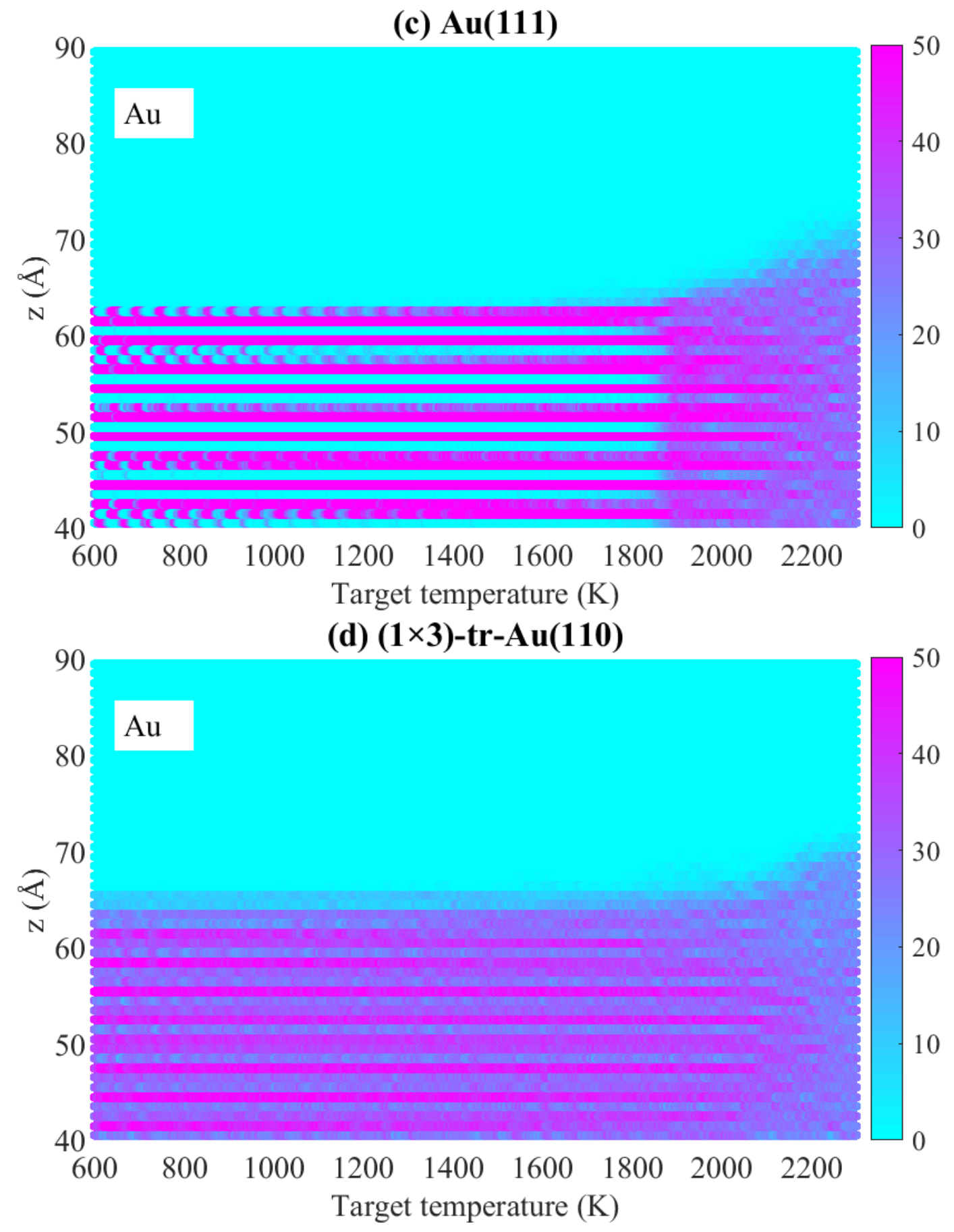


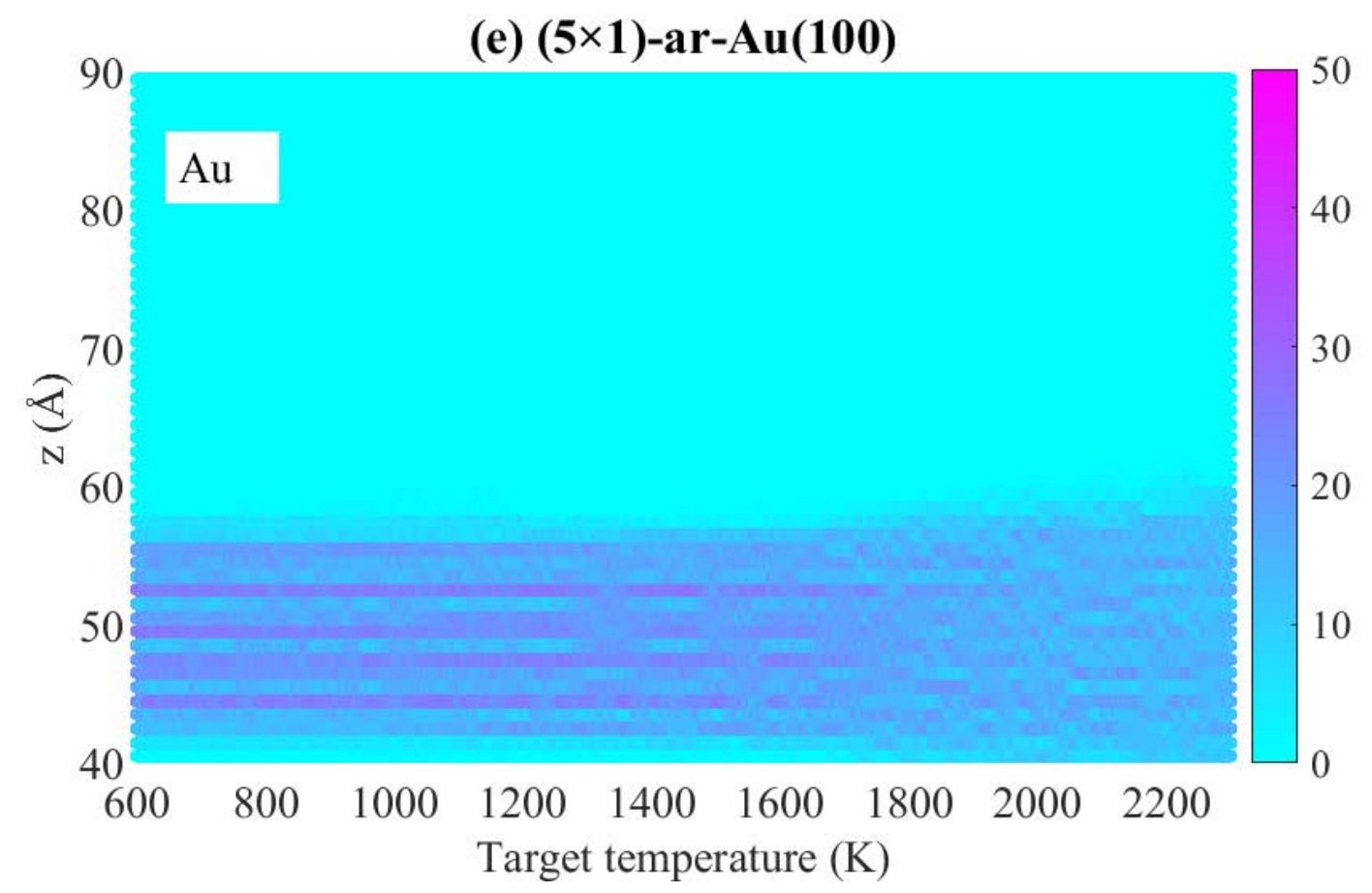


Fig. 11. Isothermal NVT simulations of $\mathrm{O}_{2}$ on the (a) (1×3)-pr-Au(110), (b) $(1 \times 2)-\mathrm{mr}-\mathrm{Au}(110)$, (c) $\mathrm{Au}(111)$, (d) $(1 \times 3)-\operatorname{tr}-\mathrm{Au}(110)$, and (e) (5×1)-ar-Au(100) surfaces. Each panel shows the laterally averaged particle density summed along the $\left[\begin{array}{lll}-1 & 1 & 0\end{array}\right]$ direction of the Au (left-hand column) and oxygen (right-hand column) for two isothermal studies performed at $1500 \mathrm{~K}$ (top row) and 2000K (bottom row). The colour scale to the right of each graph shows the laterally averaged number density in units of atoms/unit cell.
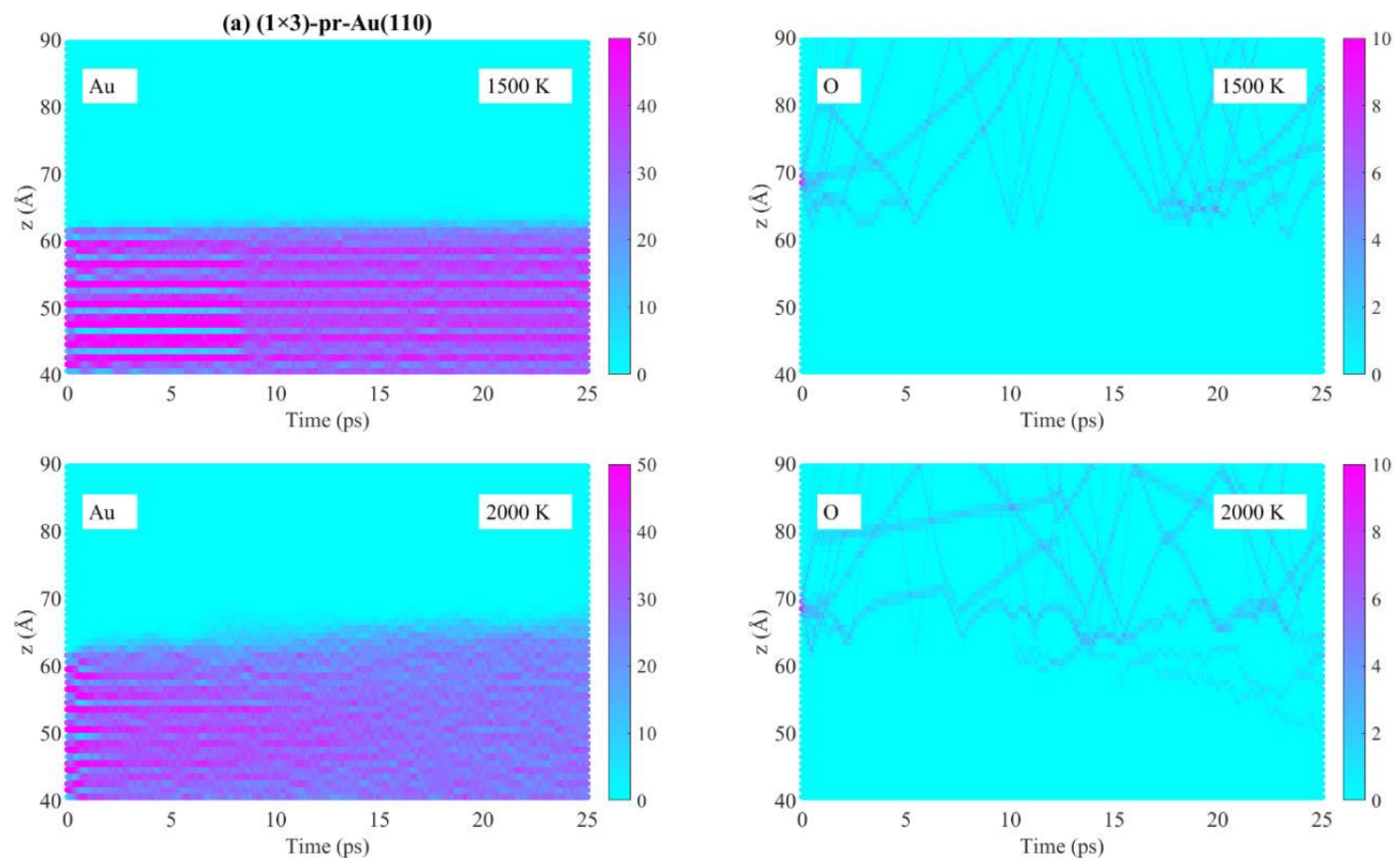

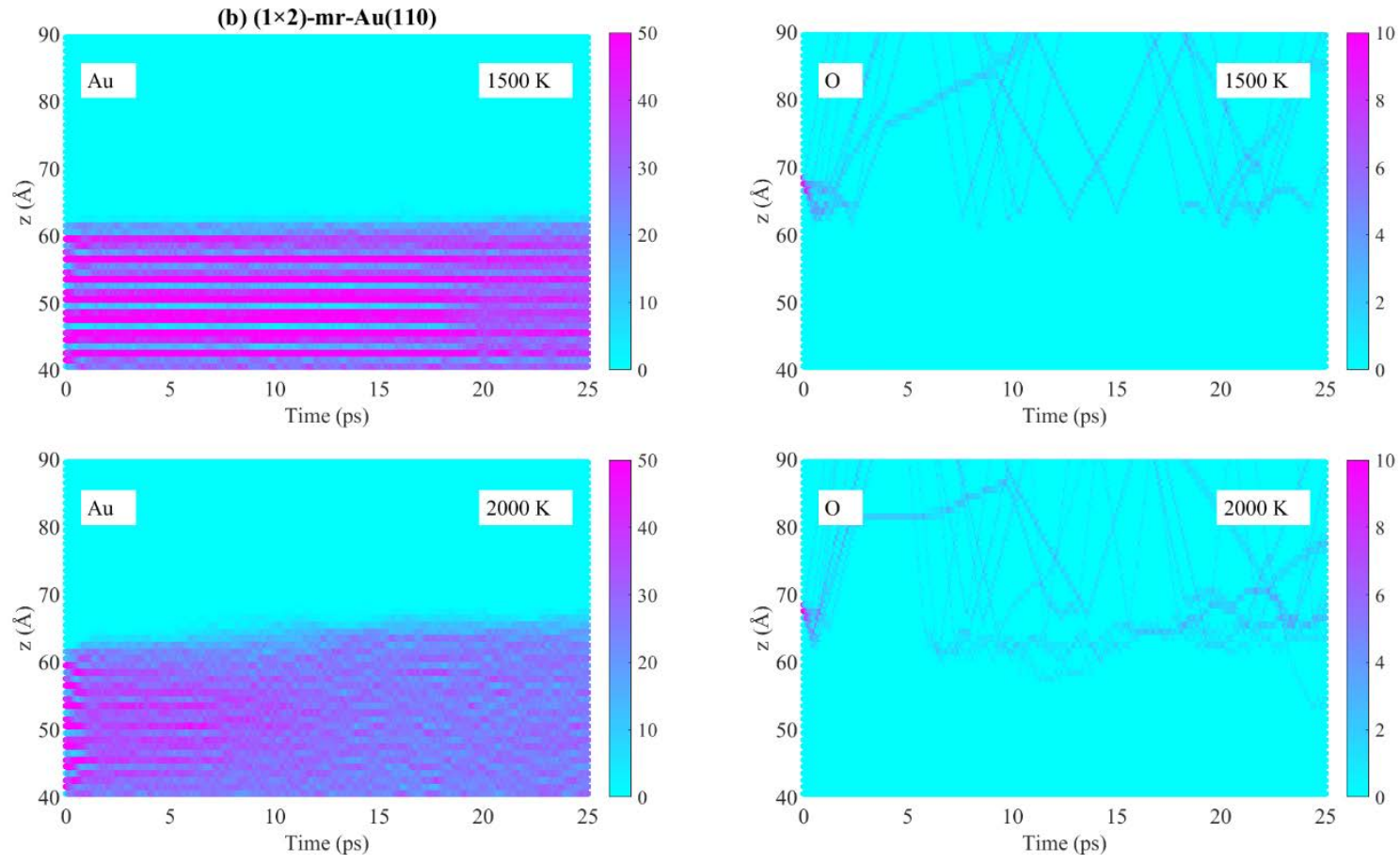

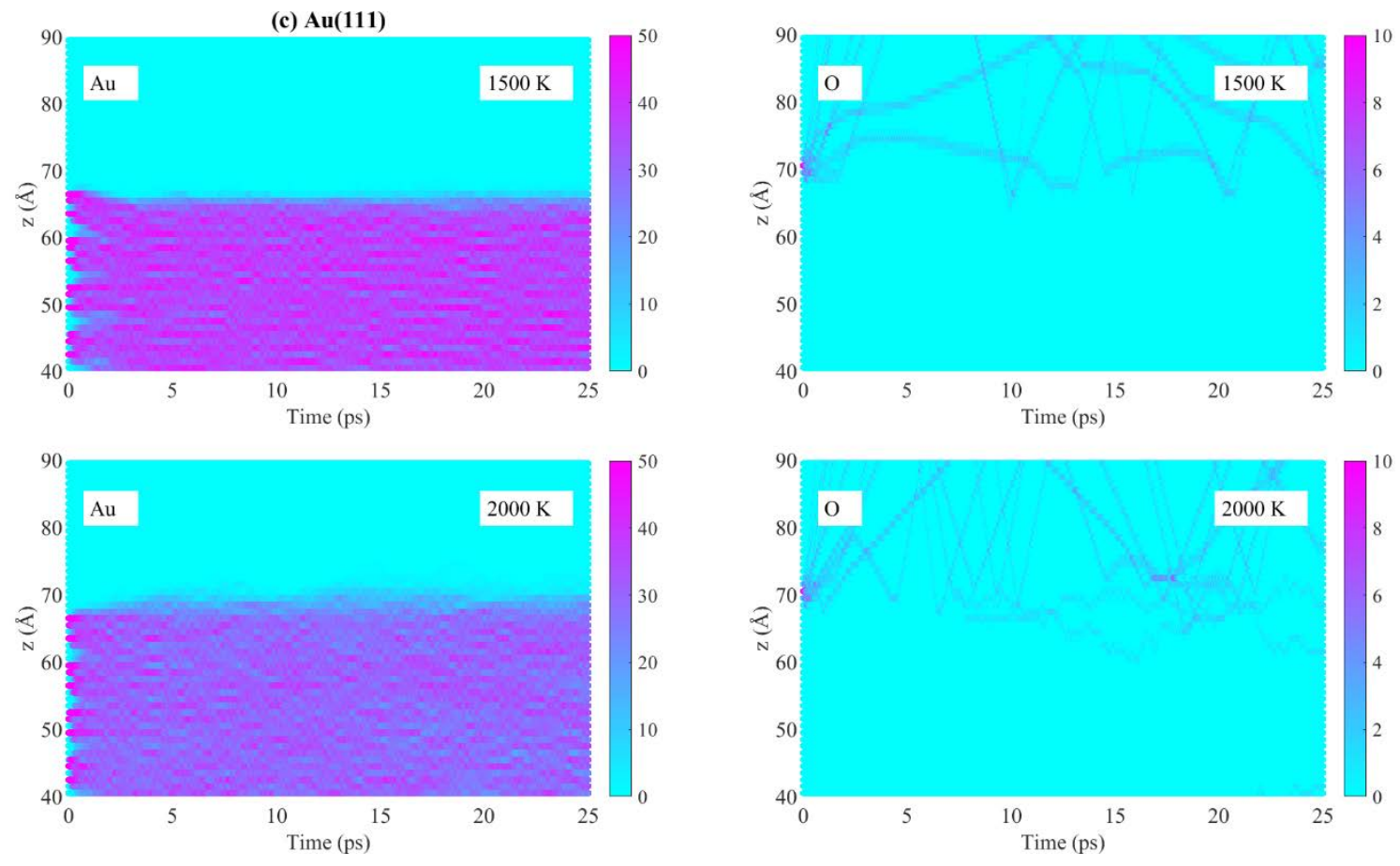

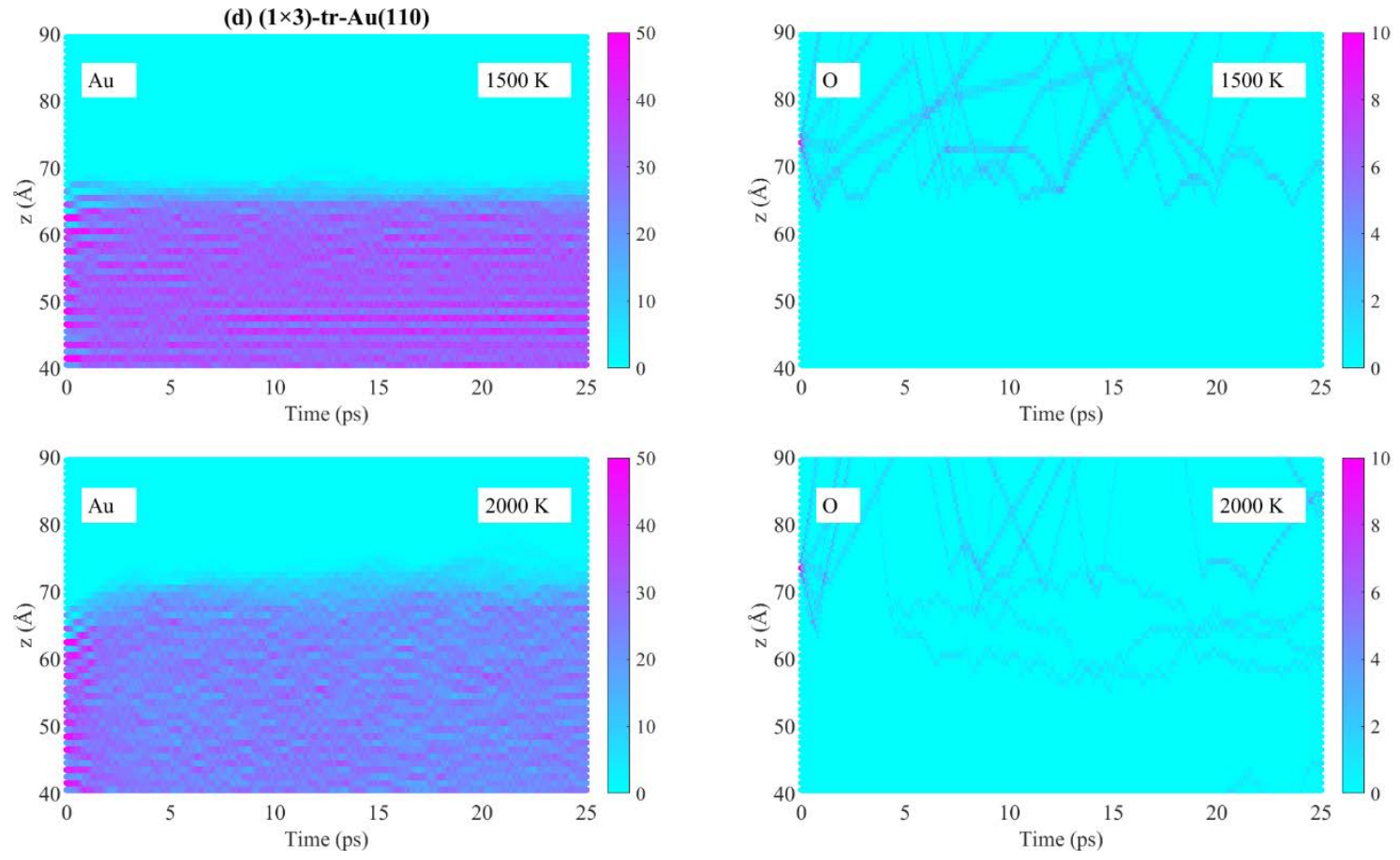

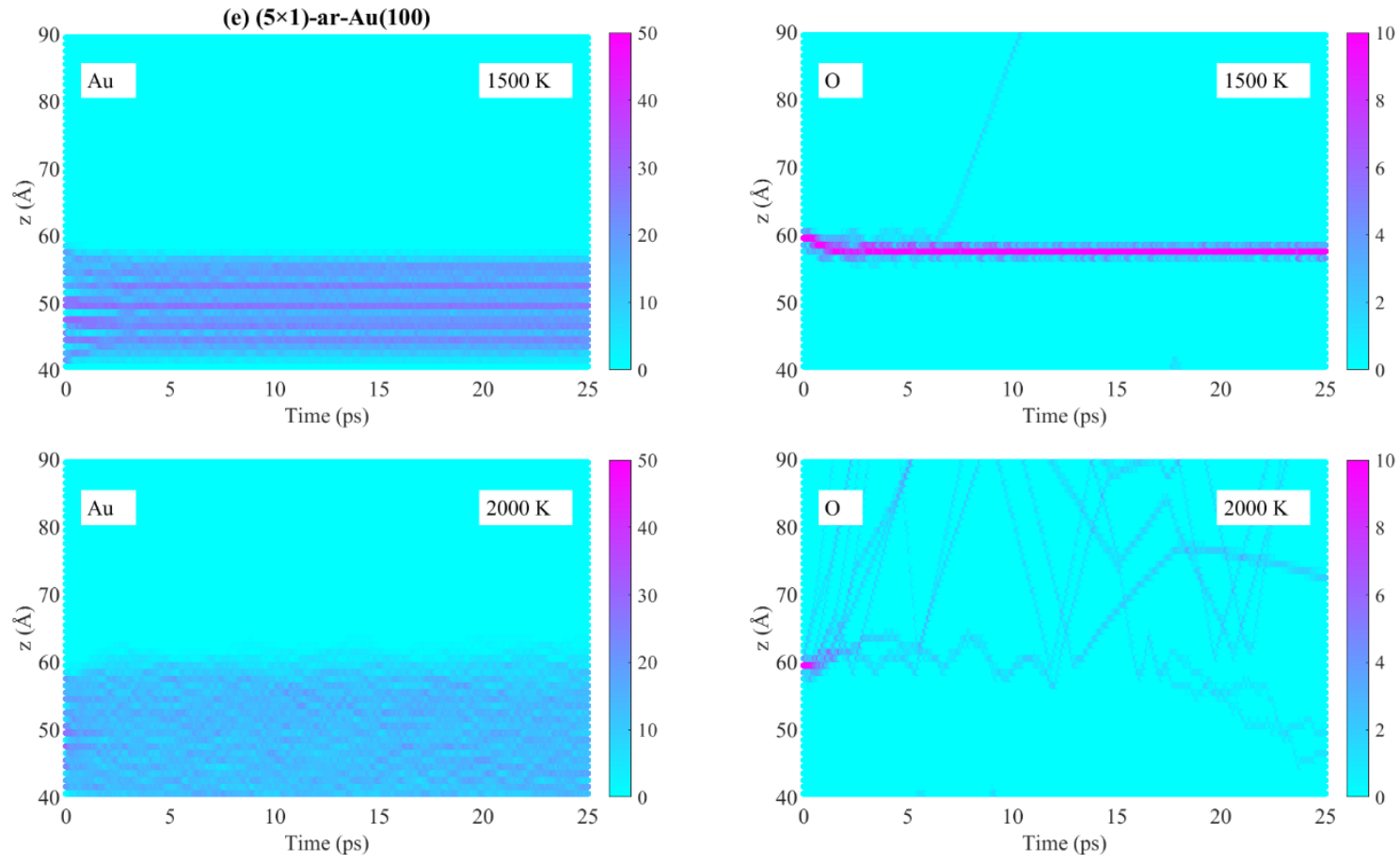
Fig. 12. Snapshots of the final structures of a grand canonical Monte Carlo (GC-MC) simulation of the oxidation of Au clusters composed of (a) 43, (b) 135 and (c) $259 \mathrm{Au}$ atoms at a temperature of $2000 \mathrm{~K}$. The captions show $\mathrm{Au}$ and $\mathrm{O}$ atoms as gold and red circles, respectively.

(a)

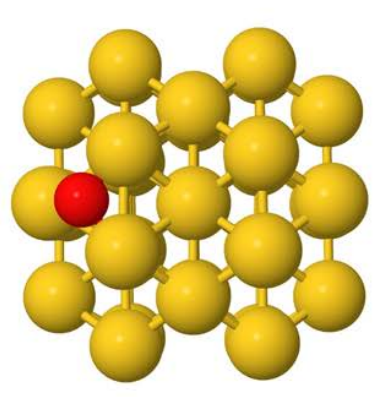

(b)

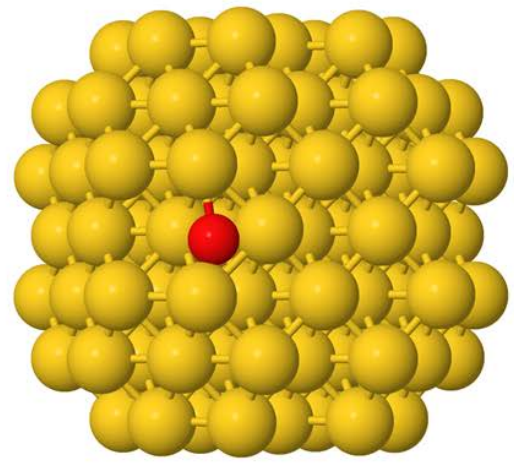

(c)

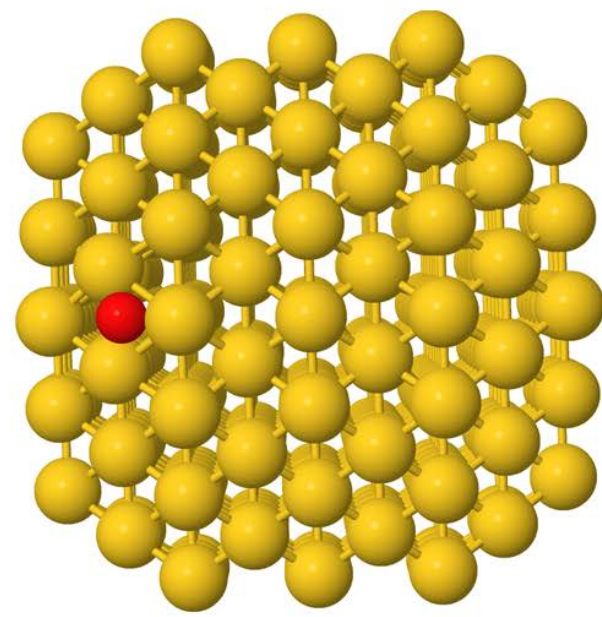




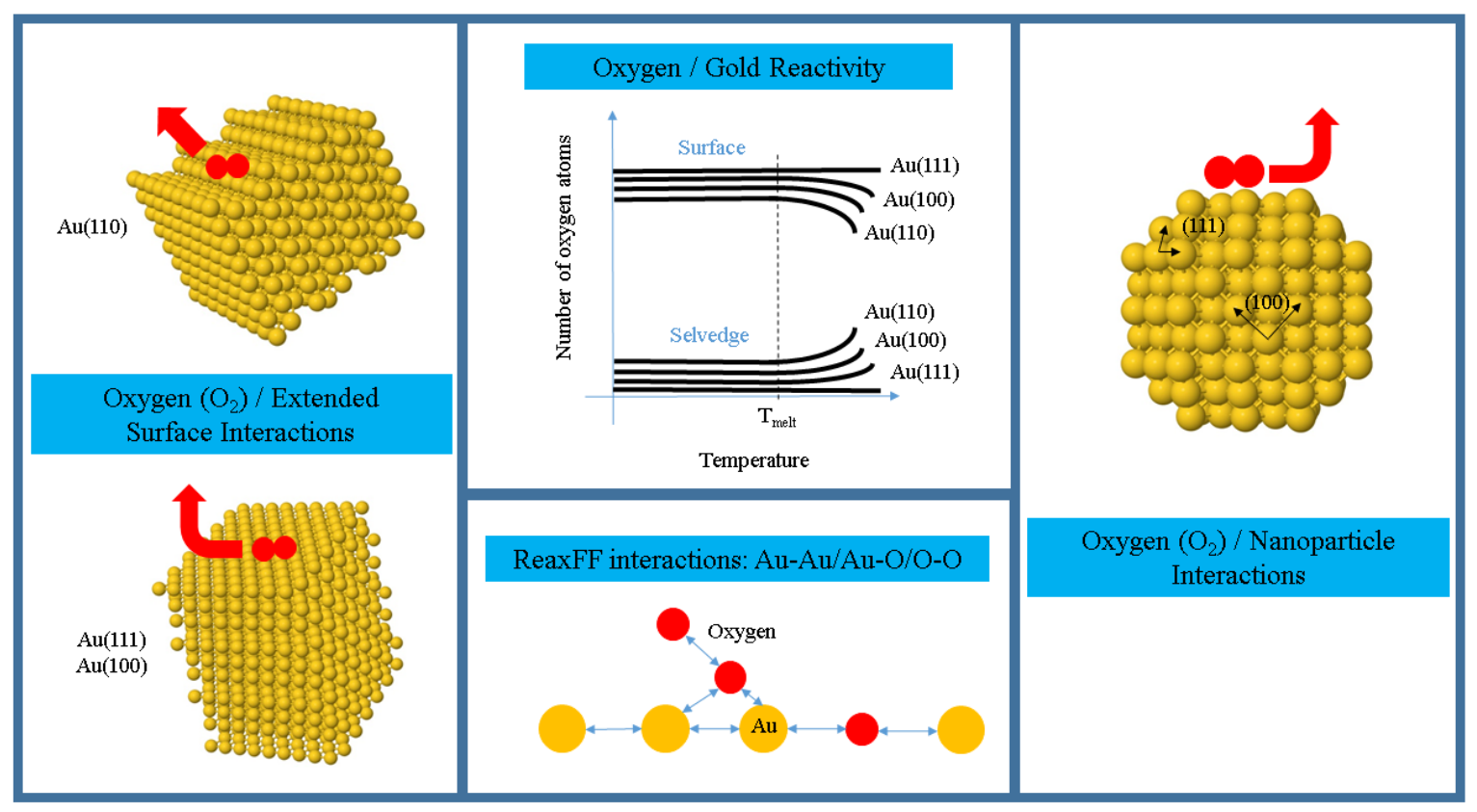

